\title{
Basins of Attraction for Two-Species Competitive Model with Quadratic Terms and the Singular Allee Effect
}

\author{
A. Brett and M. R. S. Kulenović \\ Department of Mathematics, University of Rhode Island, Kingston, RI 02881-0816, USA \\ Correspondence should be addressed to M. R. S. Kulenović; mkulenovic@mail.uri.edu
}

Received 26 August 2014; Accepted 29 October 2014

Academic Editor: Garyfalos Papashinopoulos

Copyright ( 2015 A. Brett and M. R. S. Kulenović. This is an open access article distributed under the Creative Commons Attribution License, which permits unrestricted use, distribution, and reproduction in any medium, provided the original work is properly cited.

\begin{abstract}
We consider the following system of difference equations: $x_{n+1}=x_{n}^{2} /\left(B_{1} x_{n}^{2}+C_{1} y_{n}^{2}\right), y_{n+1}=y_{n}^{2} /\left(A_{2}+B_{2} x_{n}^{2}+C_{2} y_{n}^{2}\right), n=0,1, \ldots$, where $B_{1}, C_{1}, A_{2}, B_{2}, C_{2}$ are positive constants and $x_{0}, y_{0} \geq 0$ are initial conditions. This system has interesting dynamics and it can have up to seven equilibrium points as well as a singular point at $(0,0)$, which always possesses a basin of attraction. We characterize the basins of attractions of all equilibrium points as well as the singular point at $(0,0)$ and thus describe the global dynamics of this system. Since the singular point at $(0,0)$ always possesses a basin of attraction this system exhibits Allee's effect.
\end{abstract}

\section{Introduction}

The following difference equation is known as the BevertonHolt model:

$$
x_{n+1}=\frac{a x_{n}}{1+x_{n}}, \quad n=0,1, \ldots,
$$

where $a>0$ is the rate of change (growth or decay) and $x_{n}$ is the size of the population at the $n$th generation.

This model was introduced by Beverton and Holt in 1957. It depicts density dependent recruitment of a population with limited resources which are not shared equally. The model assumes that the per capita number of offspring is inversely proportional to a linearly increasing function of the number of adults.

The Beverton-Holt model is well studied and understood and exhibits the following properties.

(a) Equation (1) has two equilibrium points 0 and $a-1$ when $a>1$.

(b) All solutions of (1) are monotonic (increasing or decreasing) sequences.

(c) If $a \leq 1$, then the zero equilibrium is a global attractor; that is, $\lim _{n \rightarrow \infty} x_{n}=0$, for all $x_{0} \geq 0$.

(d) If $a>1$, then the equilibrium point $a-1$ is a global attractor; that is, $\lim _{n \rightarrow \infty} x_{n}=a-1$, for all $x_{0}>0$. (e) Both equilibrium points are globally asymptotically stable in the corresponding regions of parameters $a \leq$ 1 and $a>1$; that is, they are global attractors with the property that small changes of initial condition $x_{0}$ result in small changes of the corresponding solution $\left\{x_{n}\right\}$.

All these properties can be derived from the explicit form of the solution of (1):

$$
\begin{gathered}
x_{n}=\frac{1}{1 /(a-1)+\left(1 / x_{0}-1 /(a-1)\right) 1 / a^{n}} \quad \text { if } a \neq 1, \\
x_{n}=\frac{1}{n+1 / x_{0}}, \quad \text { if } a=1 .
\end{gathered}
$$

See [1-3].

The following difference equation,

$$
x_{n+1}=\frac{a x_{n}^{2}}{1+x_{n}^{2}}, \quad n=0,1, \ldots,
$$

was introduced by Thomson [4] as a depensatory generalization of the Beverton-Holt stock-recruitment relationship used to develop a set of constraints designed to safeguard against overfishing; see [5] for further references. In view of 
the sigmoid shape of the function $f(u)=a u^{2} /\left(1+u^{2}\right)(3)$ is called the Sigmoid Beverton-Holt model. A very important feature of the Sigmoid Beverton-Holt model is that it exhibits the Allee effect; that is, zero equilibrium has a substantial basin of attraction, as we can see from the following results.

(a) Equation (3) has a unique zero equilibrium when $a<$ 2.

(b) Equation (3) has a zero equilibrium and the positive equilibrium $\bar{x}=1 / 2$, when $a=2$.

(c) There exist a zero equilibrium and two positive equilibria, $\bar{x}_{-}$and $\bar{x}_{+}$, when $a>2$.

(d) All solutions of (3) are monotonic (increasing or decreasing) sequences.

(e) If $a<2$, then the equilibrium point 0 is a global attractor; that is, $\lim _{n \rightarrow \infty} x_{n}=0$.

(f) If $a=2$, then the equilibrium point 0 is a global attractor, with the basin of attraction $B(0)=(0, \bar{x})$ and $\bar{x}=1 / 2$ is a nonhyperbolic equilibrium point with the basin of attraction $B(\bar{x})=[\bar{x}, \infty)$.

(g) If $a>2$, then zero equilibrium and $\bar{x}_{+}$are locally asymptotically stable, while $\bar{x}_{-}$is repeller and the basins of attraction of the equilibrium points are given as

$$
\begin{gathered}
B(0)=\left\{x_{0}: 0 \leq x_{0}<\bar{x}_{-}\right\}, \\
B\left(\bar{x}_{+}\right)=\left\{x_{0}: \bar{x}_{-}<x_{0}<\infty\right\} .
\end{gathered}
$$

In other words, the smaller positive equilibrium serves as the boundary between two basins of attraction. The zero equilibrium has the basin of attraction $B(0)$ and the model exhibits the Allee effect.

(h) The equilibrium points 0 and $\bar{x}_{+}$are globally asymptotically stable in the corresponding basins of attractions $B(0)$ and $B\left(\bar{x}_{+}\right)$.

The two dimensional analogue of (1) is the uncoupled system

$$
\begin{gathered}
x_{n+1}=\frac{a x_{n}}{1+x_{n}}, \\
y_{n+1}=\frac{b y_{n}}{1+y_{n}}, \\
n=0,1, \ldots,
\end{gathered}
$$

where $a, b$ are positive parameters. The dynamics of system (5) can be derived from dynamics of each equation. Therefore, this system has an explicit solution given by (2).

Two species can interact in several different ways through competition, cooperation, or host-parasitoid interactions. For each of these interactions, we obtain variations of system (5) all of which may require different mathematical analysis.
One such variation that exhibits competitive interaction is the following model, known as the Leslie-Gower model, which was considered in Cushing et al. [6]:

$$
\begin{array}{r}
x_{n+1}=\frac{a x_{n}}{1+x_{n}+c_{1} y_{n}}, \\
y_{n+1}=\frac{b y_{n}}{1+c_{2} x_{n}+y_{n}}, \\
n=0,1, \ldots,
\end{array}
$$

where all parameters are positive and the initial conditions are nonnegative. The global dynamics of system (6) was completed in [7]. Several variations of system (6) where the competition of two species was modeled by linear fractional difference equations were considered in [8-14]. An interesting fact is that none of these models exhibited the Allee effect.

The two dimensional analogue of system (3) is the following uncoupled system:

$$
\begin{gathered}
x_{n+1}=\frac{a x_{n}^{2}}{1+x_{n}^{2}}, \\
y_{n+1}=\frac{b y_{n}^{2}}{1+y_{n}^{2}}, \\
n=0,1, \ldots,
\end{gathered}
$$

where $a, b$ are positive parameters. The dynamics of system (7) can be derived from the dynamics of each equation in the system. Since each equation in system (7) has three possible dynamic scenarios, then system (7) possesses nine dynamic scenarios.

A variation of system (7) that exhibits competitive interactions is the system

$$
\begin{gathered}
x_{n+1}=\frac{x_{n}^{2}}{B_{1} x_{n}^{2}+C_{1} y_{n}^{2}}, \\
y_{n+1}=\frac{y_{n}^{2}}{A_{2}+B_{2} x_{n}^{2}+C_{2} y_{n}^{2}}, \\
n=0,1, \ldots,
\end{gathered}
$$

where $B_{1}, C_{1}, A_{2}, B_{2}, C_{2}>0$. This system will be considered in the remainder of this paper. We will show that system (8) has similar but more complex dynamics than system (7). We will see that like system (7) the coupled system (8) may possess $1,3,5$, or 7 equilibrium points in the hyperbolic case and 2, 4, or 6 equilibrium points in the nonhyperbolic case. In each of these cases we will show that the Allee effect is present, although $(0,0)$ is outside of the domain of definition of system (8). We will precisely describe the basins of attraction of all equilibrium points and the singular point $(0,0)$. We will show that the boundaries of the basins of attraction of the equilibrium points are the global stable manifolds of the saddle or the nonhyperbolic equilibrium points. See $[10,11$, 13-18] for related results and [19] for dynamics of competitive system with a singular point at the origin. The biological 
interpretation of a related system is given in [20,21] and similar system is treated in [22]. The specific feature of our results is that no equilibrium point in the interior of the first quadrant is computable and so our analysis is based on geometric analysis of the equilibrium curves.

\section{Preliminaries}

Our proofs use some recent general results for competitive systems of difference equations of the form:

$$
\begin{aligned}
& x_{n+1}=f\left(x_{n}, y_{n}\right), \\
& y_{n+1}=g\left(x_{n}, y_{n}\right),
\end{aligned}
$$

where $f$ and $g$ are continuous functions and $f(x, y)$ is nondecreasing in $x$ and nonincreasing in $y$ and $g(x, y)$ is nonincreasing in $x$ and nondecreasing in $y$ in some domain $A$.

Competitive systems of the form (9) were studied by many authors in $[6,7,9,13,14,23-37]$ and others.

Here we give some basic notions about monotonic maps in the plane.

We define a partial order $\preceq_{\text {se }}$ on $\mathbf{R}^{2}$ (so-called South-East ordering) so that the positive cone is the fourth quadrant; that is, this partial order is defined by

$$
\left(\begin{array}{l}
x^{1} \\
y^{1}
\end{array}\right) \preceq_{\mathrm{se}}\left(\begin{array}{l}
x^{2} \\
y^{2}
\end{array}\right) \Longleftrightarrow\left\{\begin{array}{l}
x^{1} \leqslant x^{2} \\
y^{1} \geqslant y^{2}
\end{array}\right.
$$

Similarly, we define North-East ordering as

$$
\left(\begin{array}{l}
x^{1} \\
y^{1}
\end{array}\right) \preceq_{\text {ne }}\left(\begin{array}{l}
x^{2} \\
y^{2}
\end{array}\right) \Longleftrightarrow\left\{\begin{array}{l}
x^{1} \leqslant x^{2} \\
y^{1} \leqslant y^{2} .
\end{array}\right.
$$

A map $F$ is called competitive if it is nondecreasing with respect to $\preceq_{\text {se }}$, that is, if the following holds:

$$
\left(\begin{array}{l}
x^{1} \\
y^{1}
\end{array}\right) \preceq\left(\begin{array}{l}
x^{2} \\
y^{2}
\end{array}\right) \Longrightarrow F\left(\begin{array}{l}
x^{1} \\
y^{1}
\end{array}\right) \preceq F\left(\begin{array}{l}
x^{2} \\
y^{2}
\end{array}\right) .
$$

For each $\mathbf{v}=\left(v^{1}, v^{2}\right) \in \mathbf{R}_{+}^{2}$, define $Q_{i}(\mathbf{v})$ for $i=1, \ldots, 4$ to be the usual four quadrants based on $v$ and numbered in a counterclockwise direction; for example, $Q_{1}(\mathbf{v})=\{(x, y) \in$ $\left.\mathbf{R}_{+}^{2}: v^{1} \leq x, v^{2} \leq y\right\}$.

For $S \subset R_{+}^{2}$ let $S^{\circ}$ denote the interior of $S$.

The following definition is from [35].

Definition 1. Let $R$ be a nonempty subset of $\mathbb{R}^{2}$. A competitive map $T: R \rightarrow R$ is said to satisfy condition $(O+)$ if for every $x, y$ in $R, T(x) \preceq_{\text {ne }} T(y)$ implies $x \preceq_{\text {ne }} y$, and $T$ is said to satisfy condition (O-) if for every $x, y$ in $R, T(x) \preceq_{\mathrm{ne}} T(y)$ implies $y \preceq_{\mathrm{ne}} x$.

The following theorem was proved by de Mottoni and Schiaffino [38] for the Poincaré map of a periodic competitive Lotka-Volterra system of differential equations. Smith generalized the proof to competitive and cooperative maps [34].

Theorem 2. Let $R$ be a nonempty subset of $\mathbb{R}^{2}$. If $T$ is a competitive map for which $(\mathrm{O}+)$ holds, then for all $x \in R$,
$\left\{T^{n}(x)\right\}$ is eventually componentwise monotone. If the orbit of $x$ has compact closure, then it converges to a fixed point of $T$. If instead (O-) holds, then for all $x \in R,\left\{T^{2 n}\right\}$ is eventually componentwise monotone. If the orbit of $x$ has compact closure in $R$, then its omega limit set is either a period-two orbit or a fixed point.

It is well known that a stable period-two orbit and a stable fixed point may coexist; see Hess [39].

The following result is from [35], with the domain of the map specialized to be the cartesian product of intervals of real numbers. It gives a sufficient condition for conditions $(\mathrm{O}+)$ and $(\mathrm{O}-)$.

Theorem 3. Let $R \subset \mathbb{R}^{2}$ be the cartesian product of two intervals in $\mathbb{R}$. Let $T: R \rightarrow R$ be a $C^{\prime}$ competitive map. If $T$ is injective and $\operatorname{det} J_{T}(x)>0$ for all $x \in R$ then $T$ satisfies $(O+)$. If $T$ is injective and $\operatorname{det} J_{T}(x)<0$ for all $x \in R$ then $T$ satisfies $(\mathrm{O}-)$.

Theorems 2 and 3 are quite applicable as we have shown in [40], in the case of competitive systems in the plane consisting of rational equations.

The following result is from [18], which generalizes the corresponding result for hyperbolic case from [7]. Related results have been obtained by Smith in [34].

Theorem 4. Let $\mathscr{R}$ be a rectangular subset of $\mathbb{R}^{2}$ and let $T$ be a competitive map on $\mathscr{R}$. Let $\bar{x} \in \mathscr{R}$ be a fixed point of $T$ such that $\left(Q_{1}(\bar{x}) \cup Q_{3}(\bar{x})\right) \cap \mathscr{R}$ has nonempty interior (i.e., $\bar{x}$ is not the NW or SE vertex of $\mathscr{R})$.

Suppose that the following statements are true.

(a) The map $T$ is strongly competitive on $\operatorname{int}\left(\left(Q_{1}(\bar{x}) \cup\right.\right.$ $\left.\left.Q_{3}(\bar{x})\right) \cap \mathscr{R}\right)$.

(b) $T$ is $C^{2}$ on a relative neighborhood of $\bar{x}$.

(c) The Jacobian matrix of T at $\bar{x}$ has real eigenvalues $\lambda, \mu$ such that $|\lambda|<\mu$, where $\lambda$ is stable and the eigenspace $E^{\lambda}$ associated with $\lambda$ is not a coordinate axis.

(d) Either $\lambda \geq 0$ and

$T(x) \neq \bar{x}, \quad T(x) \neq x \quad \forall x \in \operatorname{int}\left(\left(Q_{1}(\bar{x}) \cup Q_{3}(\bar{x})\right) \cap \mathscr{R}\right)$,

or $\lambda<0$ and

$T^{2}(x) \neq x \quad \forall x \in \operatorname{int}\left(\left(\mathscr{Q}_{1}(\bar{x}) \cup \mathcal{Q}_{3}(\bar{x})\right) \cap \mathscr{R}\right)$.

Then there exists a curve $\mathscr{C}$ in $\mathscr{R}$ such that

(i) $\mathscr{C}$ is invariant and a subset of $\mathscr{W}^{s}(\bar{x})$;

(ii) the endpoints of $\mathscr{C}$ lie on $\partial \mathscr{R}$;

(iii) $\bar{x} \in \mathscr{C}$;

(iv) $\mathscr{C}$ is the graph of a strictly increasing continuous function of the first variable;

(v) $\mathscr{C}$ is differentiable at $\bar{x}$ if $\bar{x} \in \operatorname{int}(\mathscr{R})$ or one sided differentiable if $\bar{x} \in \partial \mathscr{R}$, and in all cases $\mathscr{C}$ is tangential to $E^{\lambda}$ at $\bar{x}$; 
(vi) $\mathscr{C}$ separates $\mathscr{R}$ into two connected components, namely,

$$
\begin{aligned}
& \mathscr{W}_{-}:=\{x \in \mathscr{R}: \exists y \in \mathscr{C} \text { with } x \preceq y\} \\
& \mathscr{W}_{+}:=\{x \in \mathscr{R}: \exists y \in \mathscr{C} \text { with } y \preceq x\}
\end{aligned}
$$

(vii) $\mathscr{W}_{-}$is invariant, and $\operatorname{dist}\left(T^{n}(x), Q_{2}(\bar{x})\right) \rightarrow 0$ as $n \rightarrow$ $\infty$ for every $x \in \mathscr{W}_{-}$;

(viii) $\mathscr{W}_{+}$is invariant, and $\operatorname{dist}\left(T^{n}(x), \mathscr{Q}_{4}(\bar{x})\right) \rightarrow 0$ as $n \rightarrow$ $\infty$ for every $x \in \mathscr{W}_{+}$.

The following result is a direct consequence of the Trichotomy Theorem of Dancer and Hess (see [7, 39]) and is helpful for determining the basins of attraction of the equilibrium points.

Corollary 5. If the nonnegative cone of $\preceq$ is a generalized quadrant in $\mathbb{R}^{n}$, and if $T$ has no fixed points in the ordered interval $I\left(u_{1}, u_{2}\right)$ other than $u_{1}$ and $u_{2}$, then the interior of $I\left(u_{1}, u_{2}\right)$ is either a subset of the basin of attraction of $u_{1}$ or a subset of the basin of attraction of $u_{2}$.

The next results give the existence and uniqueness of invariant curves emanating from a nonhyperbolic point of unstable type, that is, a nonhyperbolic point where second eigenvalue is outside interval $[-1,1]$. Similar result for a nonhyperbolic point of stable type, that is, a nonhyperbolic point where second eigenvalue is in the interval $(-1,1)$, follows from Theorem 4. See Kulenović and Merino, Invariant Curves of Planar Competitive and Cooperative Maps.

Theorem 6. Let $\mathscr{R}=\left(a_{1}, a_{2}\right) \times\left(b_{1}, b_{2}\right)$ and let $T: \mathscr{R} \rightarrow \mathscr{R}$ be a strongly competitive map with a unique fixed point $\overline{\mathbf{x}} \in \mathscr{R}$, such that $T$ is continuously differentiable in a neighborhood of $\overline{\mathbf{x}}$. Assume further that at the point $\overline{\mathbf{x}}$ the map $T$ has associated characteristic values $\mu$ and $\nu$ satisfying $1<\mu$ and $-\mu<\nu<\mu$.

Then there exist curves $\mathscr{C}_{1}, \mathscr{C}_{2}$ in $\mathscr{R}$ and there exist $\mathbf{p}_{1}, \mathbf{p}_{2} \in$ $\partial \mathscr{R}$ with $\mathbf{p}_{1} \ll_{s e} \overline{\mathbf{x}} \ll_{s e} \mathbf{p}_{2}$ such that

(i) for $\ell=1,2, \mathscr{C}_{\ell}$ is invariant, north-east strongly linearly ordered, such that $\overline{\mathbf{x}} \in \mathscr{C}_{\ell}$ and $\mathscr{C}_{\ell} \subset Q_{3}(\overline{\mathbf{x}}) \cup Q_{1}(\overline{\mathbf{x}})$; the endpoints $\mathbf{q}_{\ell}, \mathbf{r}_{\ell}$ of $\mathscr{C}_{\ell}$, where $\mathbf{q}_{\ell} \preceq_{n e} \mathbf{r}_{\ell}$, belong to the boundary of $\mathscr{R}$. For $\ell, j \in\{1,2\}$ with $\ell \neq j, \mathscr{C}_{\ell}$ is a subset of the closure of one of the components of $\mathscr{R} \backslash \mathscr{C}_{j}$. Both $\mathscr{C}_{1}$ and $\mathscr{C}_{2}$ are tangential at $\mathbf{\overline { \mathbf { x } }}$ to the eigenspace associated with $v$;

(ii) for $\ell=1,2$, let $B_{\ell}$ be the component of $\mathscr{R} \backslash \mathscr{C}_{\ell}$ whose closure contains $\mathbf{p}_{\ell}$. Then $B_{\ell}$ is invariant. Also, for $\mathbf{x} \in$ $B_{1}, T^{n}(\mathbf{x})$ accumulates on $\mathcal{Q}_{2}\left(\mathbf{p}_{1}\right) \cap \partial \mathscr{R}$, and for $x \in B_{2}$, $T^{n}(\mathbf{x})$ accumulates on $\mathbb{Q}_{4}\left(\mathbf{p}_{2}\right) \cap \partial \mathscr{R}$.

(iii) Let $\mathscr{D}_{1}:=\mathscr{Q}_{1}(\overline{\mathbf{x}}) \cap \mathscr{R} \backslash\left(\mathscr{B}_{1} \cup \mathscr{B}_{2}\right)$ and $\mathscr{D}_{2}:=\mathbb{Q}_{3}(\overline{\mathbf{x}}) \cap$ $\mathscr{R} \backslash\left(\mathscr{B}_{1} \cup \mathscr{B}_{2}\right)$.

Then $\mathscr{D}_{1} \cup \mathscr{D}_{2}$ is invariant.
Corollary 7. Let a map $T$ with fixed point $\overline{\mathbf{x}}$ be as in Theorem 6. Let $\mathscr{D}_{1}, \mathscr{D}_{2}$ be the sets as in Theorem 6. IfT satisfies $\left(\mathrm{O}_{+}\right)$, then for $\ell=1,2, \mathscr{D}_{\ell}$ is invariant, and for every $\mathbf{x} \in$ $\mathscr{D}_{\ell}$, the iterates $T^{n}(\mathbf{x})$ converge to $\overline{\mathbf{x}}$ or to a point of $\partial \mathscr{R}$. If $T$ satisfies $\left(\mathrm{O}_{-}\right)$, then $T\left(\mathscr{D}_{1}\right) \subset \mathscr{D}_{2}$ and $T\left(\mathscr{D}_{2}\right) \subset \mathscr{D}_{1}$. For every $\mathbf{x} \in \mathscr{D}_{1} \cup \mathscr{D}_{2}$, the iterates $T^{n}(\mathbf{x})$ either converge to $\overline{\mathbf{x}}$ or converge to a period-two point or to a point of $\partial \mathscr{R}$.

\section{Local Stability of Equilibrium Points}

First we present the local stability analysis of the equilibrium points. It is interesting that the local stability analysis is the more difficult part of our analysis.

The equilibrium points of system (8) satisfy the following system of equations:

$$
\begin{gathered}
\bar{x}=\frac{\bar{x}^{2}}{B_{1} \bar{x}^{2}+C_{1} \bar{y}^{2}}, \\
\bar{y}=\frac{\bar{y}^{2}}{A_{2}+B_{2} \bar{x}^{2}+C_{2} \bar{y}^{2}}, \quad n=0,1, \ldots
\end{gathered}
$$

All solutions of system (16) with at least one zero component are given as $E_{\bar{x}}(\bar{x}, 0)$ where $\bar{x}=1 / B_{1}, E_{\bar{y}}(0, \bar{y})$ where $\bar{y}=$ $1 / 2 C_{2}$, and $E_{\bar{y}_{ \pm}}\left(0, \bar{y}_{ \pm}\right)$where $\bar{y}_{ \pm}=\left(1 \pm \sqrt{1-4 C_{2} A_{2}}\right) / 2 C_{2}$. The equilibrium point $E_{\bar{y}}(0, \bar{y})$ exists when $1=4 C_{2} A_{2}$, and $E_{\bar{y}_{+}}\left(0, \bar{y}_{ \pm}\right)$exists when $1>4 C_{2} A_{2}$.

The equilibrium points with strictly positive coordinates satisfy the following system of equations:

$$
\begin{gathered}
B_{1} x^{2}+C_{1} y^{2}-x=0, \\
A_{2}+B_{2} x^{2}+C_{2} y^{2}-y=0 .
\end{gathered}
$$

From (17) we have that all real solutions of the system (17) belong to the positive quadrant, since $B_{1} x^{2}+C_{1} y^{2}=x>0$ and $A_{2}+B_{2} x^{2}+C_{2} y^{2}=y>0$. By eliminating $y$ from (17) we obtain

$$
\begin{aligned}
& x^{4}\left(B_{2} C_{1}-B_{1} C_{2}\right)^{2}+2 C_{2} x^{3}\left(B_{2} C_{1}-B_{1} C_{2}\right) \\
& +x^{2}\left(2 A_{2} B_{2} C_{1}^{2}+B_{1}\left(C_{1}-2 A_{2} C_{1} C_{2}\right)+C_{2}^{2}\right) \\
& \quad+C_{1} x\left(2 A_{2} C_{2}-1\right)+A_{2}^{2} C_{1}^{2}=0 .
\end{aligned}
$$

The next result gives the necessary and sufficient conditions for (18) and so system (16) to have between zero and 4 solutions. As we show in Section 4.2 the global dynamics depends on the number of the equilibrium points with positive coordinates. 
Lemma 8. Let

$$
\begin{aligned}
& \Delta_{3} \\
& =16 A_{2}^{2} B_{1}^{4} C_{1}^{2}\left(1-4 A_{2} C_{2}\right)^{2}-4 B_{1}^{3} C_{1}\left(4 A_{2} C_{2}-1\right) \\
& \times\left(32 A_{2}^{3} B_{2} C_{1}^{2}-8 A_{2}^{2} C_{2}^{2}+6 A_{2} C_{2}-1\right) \\
& +B_{1}^{2}\left(256 A_{2}^{4} B_{2}^{2} C_{1}^{4}+128 A_{2}^{3} B_{2} C_{2}^{2} C_{1}^{2}\right. \\
& -8 A_{2}\left(3 B_{2} C_{1}^{2}+C_{2}^{3}\right) \\
& \left.+16 A_{2}^{2}\left(4 B_{2} C_{1}^{2} C_{2}+C_{2}^{4}\right)+C_{2}^{2}\right) \\
& +2 B_{2} B_{1} C_{1}\left(4 A _ { 2 } \left(-64 A_{2}^{2} B_{2} C_{2} C_{1}^{2}\right.\right. \\
& \left.\left.+4 A_{2}\left(3 B_{2} C_{1}^{2}+4 C_{2}^{3}\right)-13 C_{2}^{2}\right)+9 C_{2}\right) \\
& +B_{2}\left(256 A_{2}^{3} B_{2}^{2} C_{1}^{4}+B_{2} C_{1}^{2}\left(16 A_{2} C_{2}\left(9-8 A_{2} C_{2}\right)-27\right)\right. \\
& \left.+4 C_{2}^{3}\left(4 A_{2} C_{2}-1\right)\right) \text {, } \\
& \Delta_{2}=-2 B_{1}^{3} C_{1}\left(2 A_{2} C_{2}-1\right)\left(4 A_{2} C_{2}-1\right) \\
& +B_{1}^{2}\left(32 A_{2}^{2} B_{2} C_{2} C_{1}^{2}-4 A_{2}\left(3 B_{2} C_{1}^{2}+C_{2}^{3}\right)+C_{2}^{2}\right) \\
& -4 B_{2} B_{1} C_{1}\left(A_{2}\left(4 A_{2} B_{2} C_{1}^{2}+C_{2}^{2}\right)-C_{2}\right) \\
& -B_{2}\left(B_{2} C_{1}^{2}\left(9-8 A_{2} C_{2}\right)+2 C_{2}^{3}\right) \text {, } \\
& \Delta_{1}=4 A_{2} B_{1} C_{1} C_{2}-2 C_{1}\left(2 A_{2} B_{2} C_{1}+B_{1}\right)+C_{2}^{2}
\end{aligned}
$$

Assume that $B_{2} C_{1} \neq B_{1} C_{2}$. Then the following holds.

(a) If $\Delta_{3}>0, \Delta_{2}>0$, and $\Delta_{1}>0$, then (18) has four simple real roots.

(b) If $\Delta_{3}>0$ and $\Delta_{2} \leq 0 \vee\left(\Delta_{2}>0 \wedge \Delta_{1} \leq 0\right)$, then (18) has no real roots.

(c) If $\Delta_{3}<0$, then (18) has two simple real roots.

(d) If $\Delta_{3}=0$ and $\Delta_{2}<0$, then (18) has one real double root.

(e) If $\Delta_{3}=0$ and $\Delta_{2}>0$, then (18) has two real simple roots and one real double root.

(f) If $\Delta_{3}=0, \Delta_{2}=0$, and $\Delta_{1}>0$, then (18) has two real double roots.

(g) If $\Delta_{3}=0, \Delta_{2}=0$, and $\Delta_{1}<0$, then (18) has no real roots.

(h) If $\Delta_{3}=0, \Delta_{2}=0$, and $\Delta_{1}=0$, then (18) has one real root of multiplicity four.
Proof. The discrimination matrix [41] of $f(x)=A x^{4}+B x^{3}+$ $C x^{2}+D x+E$ and $f^{\prime}(x)$ is given by

$$
\begin{aligned}
& \operatorname{Discr}\left(f, f^{\prime}\right) \\
& =\left(\begin{array}{cccccccc}
A & B & C & D & E & 0 & 0 & 0 \\
0 & 4 A & 3 B & 2 C & D & 0 & 0 & 0 \\
0 & A & B & C & D & E & 0 & 0 \\
0 & 0 & 4 A & 3 B & 2 C & D & 0 & 0 \\
0 & 0 & A & B & C & D & E & 0 \\
0 & 0 & 0 & 4 A & 3 B & 2 C & D & 0 \\
0 & 0 & 0 & A & B & C & D & E \\
0 & 0 & 0 & 0 & 4 A & 3 B & 2 C & D
\end{array}\right) .
\end{aligned}
$$

Let $D_{k}$ denote the determinant of the submatrix of $\operatorname{Discr}(\tilde{f}$, $\left.\widetilde{f}^{\prime}\right)$, formed by the first $2 k$ rows and the first $2 k$ columns, for $k=1,2,3,4$ where

$$
\begin{aligned}
\tilde{f}(x)= & x^{4}\left(B_{2} C_{1}-B_{1} C_{2}\right)^{2}+2 C_{2} x^{3}\left(B_{2} C_{1}-B_{1} C_{2}\right) \\
& +x^{2}\left(2 A_{2} B_{2} C_{1}^{2}+B_{1}\left(C_{1}-2 A_{2} C_{1} C_{2}\right)+C_{2}^{2}\right) \\
& +C_{1} x\left(2 A_{2} C_{2}-1\right)+A_{2}^{2} C_{1}^{2} .
\end{aligned}
$$

So, by straightforward calculation one can see that

$$
\begin{aligned}
& D_{1}=4\left(B_{2} C_{1}-B_{1} C_{2}\right)^{4}, \\
& D_{2}=4 \Delta_{1}\left(B_{2} C_{1}-B_{1} C_{2}\right)^{6}, \\
& D_{3}=4 \Delta_{2} C_{1}^{2}\left(B_{2} C_{1}-B_{1} C_{2}\right)^{6}, \\
& D_{4}=\Delta_{3} C_{1}^{4}\left(B_{2} C_{1}-B_{1} C_{2}\right)^{6} .
\end{aligned}
$$

The rest of the proof follows in view of Theorem 1 in [41].

Geometrically solutions of system (17) are intersections of two ellipses that satisfy the equations

$$
\begin{gathered}
\frac{\left(x-1 / 2 B_{1}\right)^{2}}{1 / 4 B_{1}^{2}}+\frac{y^{2}}{1 / 4 B_{1} C_{1}}=1, \\
\frac{x^{2}}{1 / 4 B_{2} C_{2}-A_{2} / B_{2}}+\frac{\left(y-1 / 2 C_{2}\right)^{2}}{1 / 4 C_{2}^{2}-A_{2} / C_{2}}=1,
\end{gathered}
$$

with respective vertices $\left(1 / 2 B_{1}, 0\right)$ and $\left(0,1 / 2 C_{2}\right)$. See Figure 1.

Consequently when $1>4 C_{2} A_{2}$, in addition to the three equilibrium points on the axes, system (8) may have $1,2,3$, or 4 positive equilibrium points. We will refer to these equilibrium points as $E_{\mathrm{SW}}(\bar{x}, \bar{y})$ (southwest), $E_{\mathrm{SE}}(\bar{x}, \bar{y})$ (southeast), $E_{\mathrm{NW}}(\bar{x}, \bar{y})$ (northwest), and $E_{\mathrm{NE}}(\bar{x}, \bar{y})$ (northeast) where

$$
E_{\mathrm{NW}} \preceq_{\mathrm{se}} E_{\mathrm{NE}} \preceq_{\mathrm{se}} E_{\mathrm{SE}}, \quad E_{\mathrm{SW}} \preceq_{\mathrm{ne}} E_{\mathrm{NW}} .
$$

When a positive equilibrium point is nonhyperbolic we will refer to it as $E_{N}(\bar{x}, \bar{y})$. 


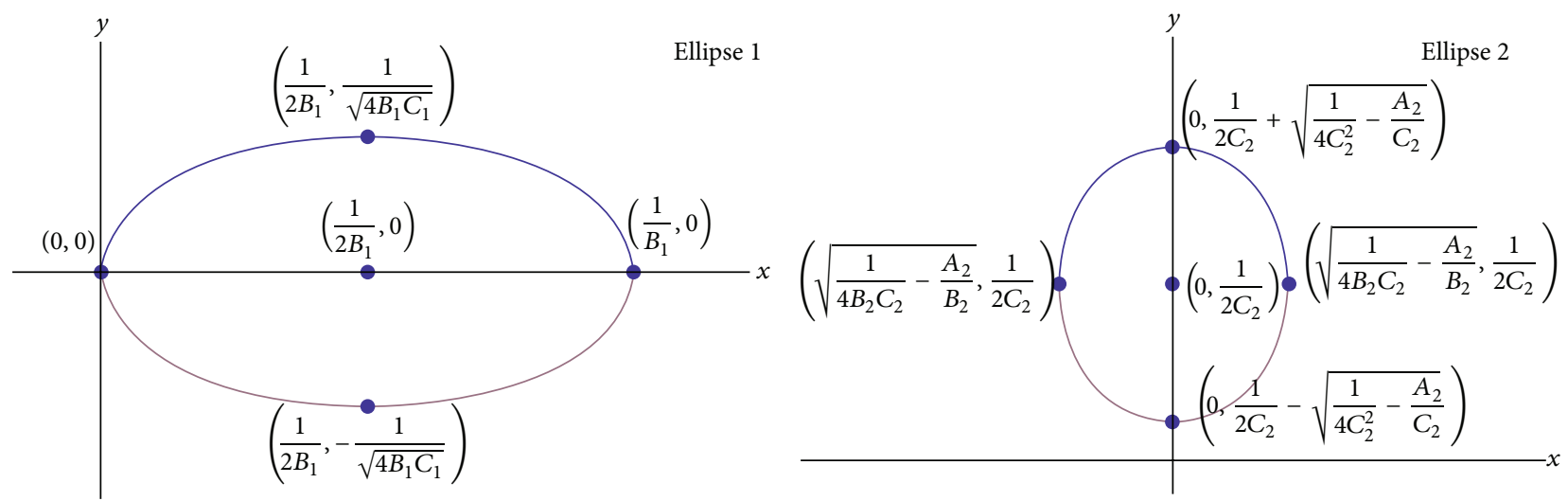

FIGURE 1: The equilibrium curves of system (8).

The map associated with system (8) has the form:

$$
T\left(\begin{array}{l}
x \\
y
\end{array}\right)=\left(\begin{array}{c}
\frac{x^{2}}{B_{1} x^{2}+C_{1} y^{2}} \\
\frac{y^{2}}{A_{2}+B_{2} x^{2}+C_{2} y^{2}}
\end{array}\right) .
$$

The Jacobian matrix of $T$ is

$$
\begin{aligned}
& J_{T}(x, y) \\
& =\left(\begin{array}{cc}
\frac{2 C_{1} x y^{2}}{\left(B_{1} x^{2}+C_{1} y^{2}\right)^{2}} & -\frac{2 C_{1} x^{2} y}{\left(B_{1} x^{2}+C_{1} y^{2}\right)^{2}} \\
-\frac{2 B_{2} x y^{2}}{\left(A_{2}+B_{2} x^{2}+C_{2} y^{2}\right)^{2}} & \frac{2 A_{2} y+2 B_{2} x^{2} y}{\left(A_{2}+B_{2} x^{2}+C_{2} y^{2}\right)^{2}}
\end{array}\right),
\end{aligned}
$$

and the Jacobian matrix of $T$ evaluated at an equilibrium $E(\bar{x}, \bar{y})$ with positive coordinates has the following form:

$$
J_{T}(\bar{x}, \bar{y})=\left(\begin{array}{cc}
\frac{2 C_{1} \bar{y}^{2}}{\bar{x}} & -2 C_{1} \bar{y} \\
-2 B_{2} \bar{x} & \frac{2 A_{2}+2 B_{2}^{2} \bar{x}}{\bar{y}}
\end{array}\right) .
$$

The determinant and trace of (27) are

$$
\begin{gathered}
\operatorname{det} J_{T}(\bar{x}, \bar{y})=\frac{4 A_{2} C_{1} \bar{y}}{\bar{x}}, \\
\operatorname{tr} J_{T}(\bar{x}, \bar{y})=\frac{2 C_{1} \bar{y}^{2}}{\bar{x}}+\frac{2 A_{2}+2 B_{2} \bar{x}^{2}}{\bar{y}} .
\end{gathered}
$$

It is worth noting that $\operatorname{det} J_{T}(\bar{x}, \bar{y})$ and $\operatorname{tr} J_{T}(\bar{x}, \bar{y})$ of (27) are both positive.

Using the equilibrium condition (17), we may rewrite the determinant and trace in the more useful form:

$$
\begin{gathered}
\operatorname{det} J_{T}(\bar{x}, \bar{y})=4 \overline{x y} B_{1} C_{2}-4 \bar{y} C_{2}-4 \bar{x} B_{1}-4 \overline{x y} B_{2} C_{1}+4, \\
\operatorname{tr} J_{T}(\bar{x}, \bar{y})=4-2 \bar{y} C_{2}-2 \bar{x} B_{1} .
\end{gathered}
$$

The characteristic equation of the matrix (27) is

$$
\lambda^{2}-\operatorname{tr} J_{T}(\bar{x}, \bar{y}) \lambda+\operatorname{det} J_{T}(\bar{x}, \bar{y})=0,
$$

whose solutions are the eigenvalues

$$
\begin{aligned}
& \lambda=\frac{\operatorname{tr} J_{T}(\bar{x}, \bar{y})-\sqrt{\left(\operatorname{tr} J_{T}(\bar{x}, \bar{y})\right)^{2}-4 \operatorname{det} J_{T}(\bar{x}, \bar{y})}}{2}, \\
& \mu=\frac{\operatorname{tr} J_{T}(\bar{x}, \bar{y})+\sqrt{\left(\operatorname{tr} J_{T}(\bar{x}, \bar{y})\right)^{2}-4 \operatorname{det} J_{T}(\bar{x}, \bar{y})}}{2} .
\end{aligned}
$$

The corresponding eigenvectors of (31) are

$$
\begin{aligned}
& E_{\lambda}=\left(\frac{1}{2 x B_{2}}\left(x B_{1}-y C_{2}+\sqrt{\left(x B_{1}-y C_{2}\right)^{2}+4 B_{2} C_{1} x y}\right), 1\right), \\
& E_{\mu} \\
& =\left(-\frac{1}{2 x B_{2}}\left(y C_{2}-x B_{1}+\sqrt{\left(x B_{1}-y C_{2}\right)^{2}+4 B_{2} C_{1} x y}\right), 1\right) .
\end{aligned}
$$

We will now consider two lemmas that will be used to prove the local stability character of the positive equilibrium points of system (8). The nonzero coordinates $(\bar{x}, \bar{y})$ of all equilibrium points will subsequently be designated with the subscripts: $r$ (repeller), $a$ (attractor), $s, s_{1}, s_{2}$ (saddlepoint), ns (nonhyperbolic of the stable type), and nu (nonhyperbolic of the unstable type).

Lemma 9. The following conditions hold for the coordinates of the positive equilibrium points, $E(\bar{x}, \bar{y})$, of system (8).

(i) For $E_{S W}\left(\bar{x}_{r}, \bar{y}_{r}\right)$ and $E_{N}\left(\bar{x}_{n u}, \bar{y}_{n u}\right)$,

$$
\bar{x}<\frac{1}{2 B_{1}}, \quad \bar{y}<\frac{1}{2 C_{2}} .
$$

(ii) For $E_{N W}\left(\bar{x}_{s_{1}}, \bar{y}_{s_{1}}\right)$,

$$
\bar{x}<\frac{1}{2 B_{1}}, \quad \bar{y}>\frac{1}{2 C_{2}} .
$$


(iii) For $E_{N E}\left(\bar{x}_{a}, \bar{y}_{a}\right), E_{N E}\left(\bar{x}_{s}, \bar{y}_{s}\right)$, and $E_{N}\left(\bar{x}_{n s}, \bar{y}_{n s}\right)$,

$$
\bar{x}>\frac{1}{2 B_{1}}, \quad \bar{y}>\frac{1}{2 C_{2}} .
$$

(iv) For $E_{S E}\left(\bar{x}_{s_{2}}, \bar{y}_{s_{2}}\right)$

$$
\bar{x}>\frac{1}{2 B_{1}}, \quad \bar{y}<\frac{1}{2 C_{2}} .
$$

Proof. This is clear from geometry. See Figure 2.

Lemma 10. The following conditions hold for the coordinates of the positive equilibrium points, $E(\bar{x}, \bar{y})$, of System (8).

(i) For $E_{S W}\left(\bar{x}_{r}, \bar{y}_{r}\right)$ and $E_{N W}\left(\bar{x}_{s_{1}}, \bar{y}_{s_{1}}\right)$,

$$
4 \overline{x y} B_{1} C_{2}-4 B_{2} C_{1} \overline{x y}+1>2 \bar{y} C_{2}+2 \bar{x} B_{1} \text {. }
$$

(ii) For $E_{N E}\left(\bar{x}_{a}, \bar{y}_{a}\right), E_{N E}\left(\bar{x}_{s}, \bar{y}_{s}\right)$, and $E_{S E}\left(\bar{x}_{s_{2}}, \bar{y}_{s_{2}}\right)$,

$$
4 \overline{x y} B_{1} C_{2}-4 B_{2} C_{1} \overline{x y}+1<2 \bar{y} C_{2}+2 \bar{x} B_{1} \text {. }
$$

(iii) For $E_{N}\left(\bar{x}_{n s}, \bar{y}_{n s}\right)$ and $E_{N}\left(\bar{x}_{n u}, \bar{y}_{n u}\right)$,

$$
4 \overline{x y} B_{1} C_{2}-4 B_{2} C_{1} \overline{x y}+1=2 \bar{y} C_{2}+2 \bar{x} B_{1} .
$$

Proof. (i) Let $m_{E 1}$ be the slope of the tangent line to ellipse $E_{1}$ at $E(\bar{x}, \bar{y})=E_{\mathrm{SW}}\left(\bar{x}_{r}, \bar{y}_{r}\right)$ and let $m_{E 2}$ be the slope of the tangent line to ellipse $E_{2}$ at $E(\bar{x}, \bar{y})=E_{\mathrm{SW}}\left(\bar{x}_{r}, \bar{y}_{r}\right)$. It is clear from geometry that

$$
m_{E 1}>m_{E 2}>0 \text {. }
$$

See Figure 2. It follows that

$$
\left.\frac{d y}{d x}\right|_{E_{1}}(\bar{x}, \bar{y})>\left.\frac{d x}{d y}\right|_{E_{2}}(\bar{x}, \bar{y})>0
$$

and in turn

$$
\frac{1-2 B_{1} \bar{x}}{2 C_{1} \bar{y}}>\frac{2 B_{2} \bar{x}}{1-2 C_{2} \bar{y}}>0
$$

Therefore

$$
4 \overline{x y} B_{1} C_{2}-4 B_{2} C_{1} \overline{x y}+1>2 \bar{y} C_{2}+2 \bar{x} B_{1} .
$$

The proofs for the remaining case in (i) and all cases in (ii) and (iii) are similar and will be omitted.

Theorem 11. The following conditions hold for the equilibrium points $E(\bar{x}, \bar{y})$ of system (8):

(i) $E_{\bar{x}}\left(\bar{x}_{a}, 0\right)$ is a locally asymptotically stable;

(ii) $E_{\bar{y}}\left(0, \bar{y}_{n s}\right)$ is nonhyperbolic of the stable type;

(iii) $E_{\bar{y}_{+}}\left(0, \bar{y}_{+a}\right)$ is locally asymptotically stable and $E_{\bar{y}_{-}}(0$, $\bar{y}_{-s}^{+}$) is a saddle point;

(iv) $E_{S W}\left(\bar{x}_{r}, \bar{y}_{r}\right)$ is a repeller; (v) $E_{N W}\left(\bar{x}_{s_{1}}, \bar{y}_{s_{1}}\right), E_{S E}\left(\bar{x}_{s_{2}}, \bar{y}_{s_{2}}\right)$, and $E_{N E}\left(\bar{x}_{s}, \bar{y}_{s}\right)$ are saddle points;

(vi) $E_{N E}\left(\bar{x}_{a}, \bar{y}_{a}\right)$ is locally asymptotically stable;

(vii) $E_{N}\left(\bar{x}_{n s}, \bar{y}_{n s}\right)$ is nonhyperbolic of the stable type;

(viii) $E_{N}\left(\bar{x}_{n u}, \bar{y}_{n u}\right)$ is nonhyperbolic of the unstable type.

Proof. (i) The eigenvalues of (26), evaluated at $E_{\bar{x}}\left(\bar{x}_{a}, 0\right)$, are $\lambda=0$ and $\mu=0$.

(ii) The eigenvalues of (26), evaluated at $E_{\bar{y}}\left(0, \bar{y}_{\mathrm{ns}}\right)$, are $\lambda=0$ and $\mu=1$ when $1=4 C_{2} A_{2}$.

(iii) The eigenvalues of (26), evaluated at $E_{\bar{y}_{+}}\left(0, \bar{y}_{+a}\right)$ and $E_{\bar{y}_{-}}\left(0, \bar{y}_{-s}\right)$, respectively, are $\lambda=0$ and $\mu_{ \pm}=2 A_{2} / \bar{y}_{ \pm}$when $1>4 C_{2} A_{2}$.

(a) Note that when $1>4 C_{2} A_{2}$,

$$
\bar{y}_{+}=\frac{1+\sqrt{1-4 C_{2} A_{2}}}{2 C_{2}}>\frac{1}{2 C_{2}}>2 A_{2} .
$$

Therefore $\mu_{+}=2 A_{2} / \bar{y}_{+}<1$.

(b) Note that when $1>4 C_{2} A_{2}, \sqrt{1-4 A_{2} C_{2}}>1-4 A_{2} C_{2}$. Therefore

$$
\mu_{-}=\frac{2 A_{2}}{\bar{y}_{-}}=\frac{4 A_{2} C_{2}}{1-\sqrt{1-4 A_{2} C_{2}}}>\frac{1-\sqrt{1-4 A_{2} C_{2}}}{1-\sqrt{1-4 A_{2} C_{2}}}=1 .
$$

In both cases, the conclusion follows.

(iv) We need to show that $\left|\operatorname{tr} J_{T}(\bar{x}, \bar{y})\right|<\left|1+\operatorname{det} J_{T}(\bar{x}, \bar{y})\right|$ and $\left|\operatorname{det} J_{T}(\bar{x}, \bar{y})\right|>1$ when $E(\bar{x}, \bar{y})=E_{\mathrm{SW}}\left(\bar{x}_{r}, \bar{y}_{r}\right)$. Since $\operatorname{tr} J_{T}(\bar{x}, \bar{y})$ and det $J_{T}(\bar{x}, \bar{y})$ are both positive, our conditions become $\operatorname{tr} J_{T}(\bar{x}, \bar{y})<1+\operatorname{det} J_{T}(\bar{x}, \bar{y})$ and $\operatorname{det} J_{T}(\bar{x}, \bar{y})>1$. We will first show that det $J_{T}(\bar{x}, \bar{y})>1$. By (37) we have

$$
\begin{aligned}
\operatorname{det} & J_{T}(\overline{x y})-1 \\
& =4 \overline{x y} B_{1} C_{2}-4 \overline{x y} B_{2} C_{1}-4 \bar{y} C_{2}-4 \bar{x} B_{1}+4-1 \\
& >2 \bar{y} C_{2}+2 \bar{x} B_{1}-1-4 \bar{y} C_{2}-4 \bar{x} B_{1}+4-1 \\
& =1-2 \bar{y} C_{2}+1-2 \bar{x} B_{1} .
\end{aligned}
$$

By (33) we have $1-2 \bar{y} C_{2}+1-2 \bar{x} B_{1}>0$.

Therefore $\operatorname{det} J_{T}(\bar{x}, \bar{y})>1$. We will next show that $\operatorname{tr} J_{T}(\bar{x}$, $\bar{y})<1+\operatorname{det} J_{T}(\bar{x}, \bar{y})$.

By (37) we have

$$
\begin{aligned}
1+ & \operatorname{det} J_{T}(\bar{x}, \bar{y})-\operatorname{tr} J_{T}(\bar{x}, \bar{y}) \\
= & 1+\left(4 \overline{x y} B_{1} C_{2}-4 \bar{y} C_{2}-4 \bar{x} B_{1}-4 \overline{x y} B_{2} C_{1}+4\right) \\
& -\left(4-2 \bar{y} C_{2}-2 \bar{x} B_{1}\right) \\
= & 4 \overline{x y} B_{1} C_{2}-4 \overline{x y} B_{2} C_{1}+1-2 \bar{y} C_{2}-2 \bar{x} B_{1} \\
> & 2 \bar{y} C_{2}+2 \bar{x} B_{1}-2 \bar{y} C_{2}-2 \bar{x} B_{1}=0 .
\end{aligned}
$$

Therefore $\operatorname{tr} J_{T}(\bar{x}, \bar{y})<1+\operatorname{det} J_{T}(\bar{x}, \bar{y})$. 

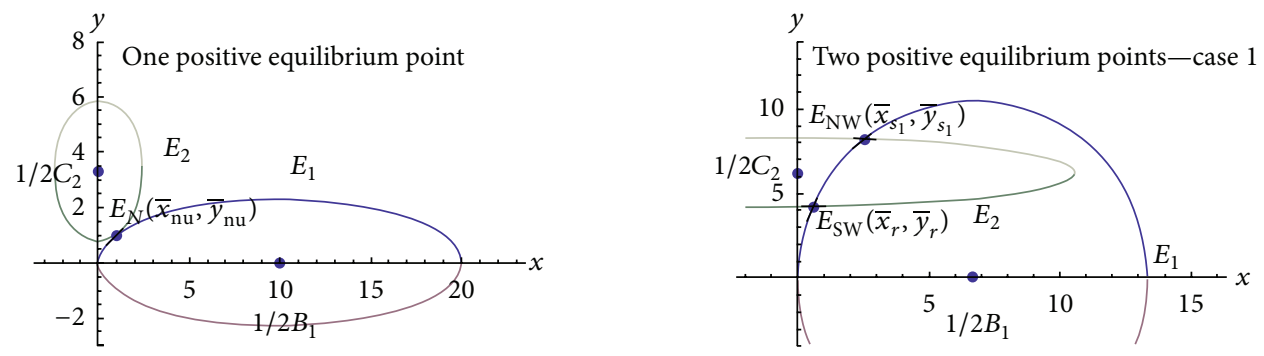

$B_{1}=5 / 100, C_{1}=95 / 100, A_{2}=13 / 19, B_{2}=63 / 380$,

$$
B_{1}=0.075, C_{1}=0.03, A_{2}=2.79, B_{2}=0.003 \text {, }
$$

$C_{2}=15 / 100$

$C_{2}=0.08$
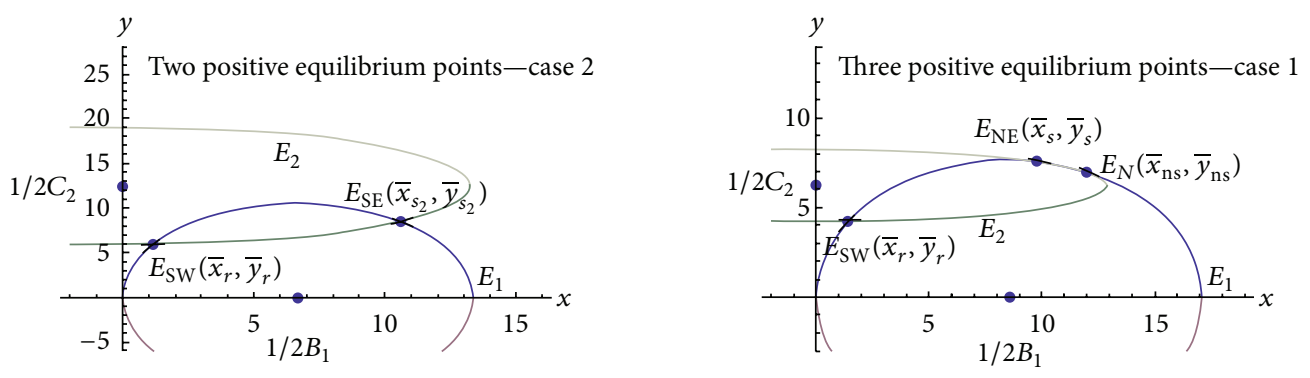

$B_{1}=0.075, C_{1}=0.03, A_{2}=4.5, B_{2}=0.01$,

$B_{1}=83 / 1416, C_{1}=30 / 413, A_{2}=349 / 125, B_{2}=2 / 1000$,

$C_{2}=0.04$

$C_{2}=8 / 100$
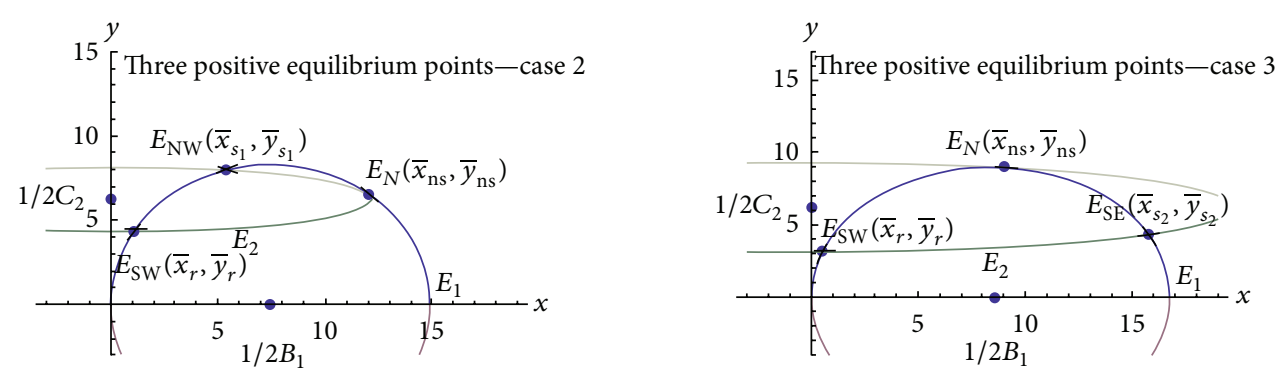

$B_{1}=317 / 4728, C_{1}=350 / 6501, A_{2}=1767 / 625, B_{2}=2 / 1000$,

$C_{2}=8 / 100$

$B_{1}=64 / 1071, C_{1}=55 / 1071, A_{2}=1179 / 500, B_{2}=2 / 1000$,

$C_{2}=8 / 100$

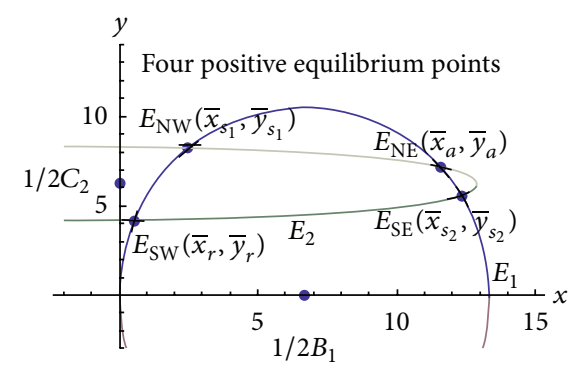

$B_{1}=0.075, C_{1}=0.03, A_{2}=2.79, B_{2}=0.002$,
$C_{2}=0.08$

FIGURE 2: Local stability. 
(v) We need to show that $|\operatorname{tr} J(\bar{x}, \bar{y})|>\left|1+\operatorname{det} J_{T}(\bar{x}, \bar{y})\right|$ when $E(\bar{x}, \bar{y})=E_{\mathrm{NW}}\left(\bar{x}_{s_{1}}, \bar{y}_{s_{1}}\right)$. Since $\operatorname{tr} J_{T}(\bar{x}, \bar{y})$ and $\operatorname{det} J_{T}(\bar{x}$, $\bar{y})$ are both positive, our condition becomes $\operatorname{tr} J_{T}(\bar{x}, \bar{y})>1+$ $\operatorname{det} J_{T}(\bar{x}, \bar{y})$. By $(37)$ we have

$$
\begin{aligned}
& \operatorname{tr} J_{T}(\bar{x}, \bar{y})-\left(1+\operatorname{det} J_{T}(\bar{x}, \bar{y})\right) \\
&=4-2 \bar{y} C_{2}-2 \bar{x} B_{1} \\
& \quad-\left(1+4 \overline{x y} B_{1} C_{2}-4 \bar{y} C_{2}-4 \bar{x} B_{1}-4 \overline{x y} B_{2} C_{1}+4\right) \\
&=2 \bar{x} B_{1}+2 \bar{y} C_{2}-4 \overline{x y} B_{1} C_{2}+4 \overline{x y} B_{2} C_{1}-1 \\
&> 4 \overline{x y} B_{1} C_{2}-4 B_{2} C_{1} \overline{x y}+1-4 \overline{x y} B_{1} C_{2}+4 \overline{x y} B_{2} C_{1}-1 .
\end{aligned}
$$

Therefore $\operatorname{tr} J_{T}(\bar{x}, \bar{y})>1+\operatorname{det} J_{T}(\bar{x}, \bar{y})$. The proofs that $E_{\mathrm{SE}}\left(\bar{x}_{s_{2}}, \bar{y}_{s_{2}}\right)$ and $E_{\mathrm{NE}}\left(\bar{x}_{s}, \bar{y}_{s}\right)$ are saddle points are similar and will be omitted.

(vi) We need to show that $\left|\operatorname{tr} J_{T}(\bar{x}, \bar{y})\right|<1+\operatorname{det} J_{T}(\bar{x}, \bar{y})$ and $\operatorname{det} J_{T}(\bar{x}, \bar{y})<1$ when $E(\bar{x}, \bar{y})=E_{\mathrm{NE}}\left(\bar{x}_{a}, \bar{y}_{a}\right)$. Since $\operatorname{tr} J_{T}(\bar{x}, \bar{y})$ and $\operatorname{det} J_{T}(\bar{x}, \bar{y})$ are both positive, our conditions become $\operatorname{tr} J_{T}(\bar{x}, \bar{y})<1+\operatorname{det} J_{T}(\bar{x}, \bar{y})$ and $\operatorname{det} J_{T}(\bar{x}, \bar{y})<1$. We will first show that $\operatorname{det} J_{T}(\bar{x}, \bar{y})<1$. By (38) we have

$$
\begin{aligned}
\operatorname{det} & J_{T}(\bar{x}, \bar{y})-1 \\
= & \left(4 \overline{x y} B_{1} C_{2}-4 \bar{y} C_{2}-4 \bar{x} B_{1}-4 \overline{x y} B_{2} C_{1}+4\right)-1 \\
= & 4 \overline{x y} B_{1} C_{2}-4 \overline{x y} B_{2} C_{1}-4 \bar{y} C_{2}-4 \bar{x} B_{1}+3 \\
& <2 \bar{y} C_{2}+2 \bar{x} B_{1}-1-4 \bar{y} C_{2}-4 \bar{x} B_{1}+3 \\
& =1-2 \bar{y} C_{2}+1-2 \bar{x} B_{1} .
\end{aligned}
$$

By (35) we have $1-2 \bar{y} C_{2}+1-2 \bar{x} B_{1}<0$.

Therefore $\operatorname{det} J_{T}(\bar{x}, \bar{y})<1$. We will next show that $\operatorname{tr} J_{T}(\bar{x}, \bar{y})<1+\operatorname{det} J_{T}(\bar{x}, \bar{y})$. By (38) we have

$$
\begin{aligned}
& 1+\operatorname{det} J_{T}(\bar{x}, \bar{y})-\operatorname{tr} J_{T}(\bar{x}, \bar{y}) \\
&=\left(1+4 \bar{x} \bar{y} B_{1} C_{2}-4 \bar{y} C_{2}-4 \bar{x} B_{1}-4 \overline{x y} B_{2} C_{1}+4\right) \\
& \quad-\left(4-2 \bar{y} C_{2}-2 \bar{x} B_{1}\right) \\
&=4 \overline{x y} B_{1} C_{2}-4 \overline{x y} B_{2} C_{1}+1-2 \bar{y} C_{2}-2 \bar{x} B_{1} \\
&> 2 \bar{y} C_{2}+2 \bar{x} B_{1}-2 \bar{y} C_{2}-2 \bar{x} B_{1} .
\end{aligned}
$$

Therefore $\operatorname{tr} J_{T}(\bar{x}, \bar{y})<1+\operatorname{det} J_{T}(\bar{x}, \bar{y})$.

(vii) By (29) and (31) we have

$$
\begin{aligned}
\lambda= & \left(4-2 y C_{2}-2 x B_{1}\right) \\
& -\left(\left(4-2 y C_{2}-2 x B_{1}\right)^{2}\right. \\
& \left.\left.\quad-4\left(4 x y B_{1} C_{2}-4 y C_{2}-4 x B_{1}-4 x y B_{2} C_{1}+4\right)\right)^{1 / 2}\right) \\
& \quad \times(2)^{-1},
\end{aligned}
$$

$$
\begin{aligned}
& \mu=\left(\left(4-2 y C_{2}-2 x B_{1}\right)\right. \\
& +\left(\left(4-2 y C_{2}-2 x B_{1}\right)^{2}\right. \\
& \left.\left.\quad \quad-4\left(4 x y B_{1} C_{2}-4 y C_{2}-4 x B_{1}-4 x y B_{2} C_{1}+4\right)\right)^{1 / 2}\right) \\
& \quad \times(2)^{-1} .
\end{aligned}
$$

By (39), we have $\lambda=3-2 \bar{y} C_{2}-2 \bar{x} B_{1}$ and $\mu=1$. By (35), we have $\lambda<1$. The conclusion follows.

(viii) The proof of (viii) is similar to the proof of (vii) and will be omitted.

\section{Global Results}

In this section we combine the results from Sections 2 and 3 to prove the global results for system (8). First, we present the behavior of the solutions of system (8) on coordinate axes and then we prove that the map $T$ which corresponds to system (8) is injective and that it satisfies $(\mathrm{O}+)$.

4.1. Convergence of Solutions on the Coordinate Axes: Injectivity and $(\mathrm{O}+)$. When $y_{n}=0$, system (8) becomes

$$
x_{n+1}=\frac{1}{B_{1}}, \quad y_{n+1}=0, \quad n=0,1, \ldots
$$

When $x_{n}=0$, system (8) becomes

$$
x_{n+1}=0, \quad y_{n+1}=\frac{y_{n}^{2}}{A_{2}+C_{2} y_{n}^{2}}, \quad n=0,1, \ldots
$$

It follows from (52) and (53) that solutions of system (8) with initial conditions on the $x$-axis remain on the $x$-axis and solutions of system (8) with initial conditions on the $y$-axis remain on the $y$-axis.

Theorem 12. The following conditions hold for solutions $\left\{\left(x_{n}\right.\right.$, $\left.\left.y_{n}\right)\right\}$ of system (8) with initial conditions on the $x$ or $y$-axis.

(i) $E_{\bar{x}}\left(\bar{x}_{a}, 0\right)$ is a superattractor of all solutions $\left\{\left(x_{n}, y_{n}\right)\right\}$ of system (8) with initial conditions on the $x$-axis.

(ii) When no equilibrium points exist on the $y$ axis, if $x_{0}=$ 0 , then $\lim _{n \rightarrow \infty}\left(x_{n}, y_{n}\right)=(0,0)$.

(iii) When $E_{\bar{y}}\left(0, \bar{y}_{n s}\right)$ exists,

(a) if $x_{0}=0$ and $y_{0}>\bar{y}_{n s}$, then $\lim _{n \rightarrow \infty}\left(x_{n}, y_{n}\right)=$ $\left(0, \bar{y}_{n s}\right)$

(b) if $x_{0}=0$ and $0<y_{0}<\bar{y}_{n s}$, then $\lim _{n \rightarrow \infty}\left(x_{n}\right.$, $\left.y_{n}\right)=(0,0)$.

(iv) When $E_{\bar{y}_{+}}\left(0, \bar{y}_{+a}\right)$ and $E_{\bar{y}_{-}}\left(0, \bar{y}_{-s}\right)$ exist,

(a) if $x_{0}=0$ and $y_{0}>\bar{y}_{+a}$, then $\lim _{n \rightarrow \infty}\left(x_{n}, y_{n}\right)=$ $\left(0, \bar{y}_{+a}\right)$; 
(b) if $x_{0}=0$ and $\bar{y}_{-s}<y_{0}<\bar{y}_{+a}$, then $\lim _{n \rightarrow \infty}\left(x_{n}\right.$, $\left.y_{n}\right)=\left(0, \bar{y}_{+a}\right)$

(c) if $x_{0}=0$ and $0<y_{0}<\bar{y}_{-s}$, then $\lim _{n \rightarrow \infty}\left(x_{n}\right.$, $\left.y_{n}\right)=(0,0)$.

Proof. (i) When $y_{0}=0$, it follows directly from (52) that $\left(x_{n}, y_{n}\right)=\left(\bar{x}_{a}, 0\right)$ for $n>1$.

(ii) In this case $1<4 A_{2} C_{2}$. By (53) it can be shown that

$$
y_{n+1}-y_{n}=\frac{-y_{n}\left(C_{2}\left(y_{n}-1 / 2 C_{2}\right)^{2}+A_{2}-1 / 4 C_{2}\right)}{A_{2}+C_{2} y_{n}^{2}} .
$$

By (54), when $1<4 A_{2} C_{2}$, it is clear that $\left\{y_{n}\right\}$ is a stricly decreasing sequence, and so is convergent. It follows that $\left\{y_{n}\right\}$ converges to 0 .

(iii) In this case, $1=4 A_{2} C_{2}$, and we may rewrite (54) as

$$
y_{n+1}-y_{n}=\frac{-y_{n}\left(C_{2}\left(y_{n}-\bar{y}_{\mathrm{ns}}\right)^{2}\right)}{A_{2}+C_{2} y_{n}^{2}} .
$$

By (55) it is clear that $\left\{y_{n}\right\}$ is a stricly decreasing sequence, and so is convergent. It follows that $\left\{y_{n}\right\}$ converges to $\bar{y}_{\text {ns }}$ when $y_{0}>\bar{y}_{n s}$, and $\left\{y_{n}\right\}$ converges to 0 when $0<y_{0}<\bar{y}_{n s}$.

(iv) In this case, $1>4 A_{2} C_{2}$. By (53), it can be shown that

$$
y_{n+1}-y_{n}=\frac{-C_{2} y_{n}\left(y_{n}-\bar{y}_{+a}\right)\left(y_{n}-\bar{y}_{-s}\right)}{A_{2}+C_{2} y_{n}^{2}} .
$$

By (56), it is clear that $\left\{y_{n}\right\}$ is a stricly decreasing sequence (and so is convergent) when $y_{0}>\bar{y}_{+a}$ and when $0<y_{0}<$ $\bar{y}_{-s}$, and a strictly increasing sequence (and so is convergent) when $\bar{y}_{-s}<y_{0}<\bar{y}_{+a}$. It follows that $\left\{y_{n}\right\}$ converges to $\bar{y}_{+a}$ when $y_{0}>\bar{y}_{+a}$ and when $\bar{y}_{-s}<y_{0}<\bar{y}_{+a}$ and converges to 0 when $0<y_{0}<\bar{y}_{-s}$.

Theorem 13. The map $T$ which corresponds to system (8) is injective.

Proof. Indeed,

$$
\begin{aligned}
T\left(\begin{array}{l}
x_{1} \\
y_{1}
\end{array}\right) & =T\left(\begin{array}{l}
x_{2} \\
y_{2}
\end{array}\right) \Longleftrightarrow\left(\begin{array}{c}
\frac{x_{1}^{2}}{B_{1} x_{1}^{2}+C_{1} y_{1}^{2}} \\
\frac{y_{1}^{2}}{A_{2}+B_{2} x_{1}^{2}+C_{2} y_{1}^{2}}
\end{array}\right) \\
& =\left(\begin{array}{c}
\frac{x_{2}^{2}}{B_{1} x_{2}^{2}+C_{1} y_{2}^{2}} \\
\frac{y_{2}^{2}}{A_{2}+B_{2} x_{2}^{2}+C_{2} y_{2}^{2}}
\end{array}\right)
\end{aligned}
$$

which is equivalent to

$$
y_{2}^{2} x_{1}^{2}=y_{1}^{2} x_{2}^{2}, \quad y_{1}=y_{2} .
$$

This immediatly implies $x_{1}=x_{2}$.

Theorem 14. The map $T$ which corresponds to system (8) satisfies $\left(\mathrm{O}^{+}\right)$. All solutions of system (8) converge to either an equilibrium point or to $(0,0)$.
Proof. Assume that

$$
\begin{aligned}
T\left(\begin{array}{l}
x_{1} \\
y_{1}
\end{array}\right) & \leq_{\mathrm{ne}} T\left(\begin{array}{l}
x_{2} \\
y_{2}
\end{array}\right) \Longrightarrow\left(\begin{array}{c}
\frac{x_{1}^{2}}{B_{1} x_{1}^{2}+C_{1} y_{1}^{2}} \\
\frac{y_{1}^{2}}{A_{2}+B_{2} x_{1}^{2}+C_{2} y_{1}^{2}}
\end{array}\right) \\
& \leq_{\mathrm{ne}}\left(\begin{array}{c}
\frac{x_{2}^{2}}{B_{1} x_{2}^{2}+C_{1} y_{2}^{2}} \\
\frac{y_{2}^{2}}{A_{2}+B_{2} x_{2}^{2}+C_{2} y_{2}^{2}}
\end{array}\right) .
\end{aligned}
$$

The last inequality is equivalent to

$$
y_{2}^{2} x_{1}^{2} \leq y_{1}^{2} x_{2}^{2}, \quad y_{1} \leq y_{2}
$$

Suppose $x_{2}<x_{1}$. Then $y_{1}^{2} x_{2}^{2}<y_{2}^{2} x_{1}^{2}$, which contradicts (60). Consequently $x_{1} \leq x_{2}$ and so $\left(\begin{array}{l}x_{1} \\ y_{1}\end{array}\right) \leq_{\text {ne }}\left(\begin{array}{l}x_{2} \\ y_{2}\end{array}\right)$.

Thus we conclude that all solutions of system (8) are eventually monotonic for all values of parameters. Furthermore it is clear that all solutions are bounded. Indeed every solution of (8) satisfies

$$
x_{n} \leq \frac{1}{B_{1}}, \quad y_{n} \leq \frac{1}{C_{2}} .
$$

Consequently, all solutions of system (8) converge to an equilibrium point or to $(0,0)$.

4.2. Global Dynamics. In this section we show that there are seven dynamic scenarios for global dynamics of system (8). See Figures 3 and 4 for geometric interpretations of these scenarios.

Theorem 15. Assume that $1<4 A_{2} C_{2}$. Then system (8) has one equilibrium point $E_{\bar{x}}$ which is locally asymptotically stable. The singular point $E_{0}(0,0)$ is global attractor of all points on $y$ axis and every point on $x$-axis is attracted to $E_{\bar{x}}$. Furthermore, every point in the interior of the first quadrant is attracted to $E_{0}$ or $E_{\bar{x}}$.

Proof. Local stability of all equilibrium points follows from Theorem 11. In view of Theorem 12, every solution that starts on the $y$-axis converges to 0 in a decreasing manner and every solution that starts on $x$-axis is equal to $E_{\bar{x}}$ in a single step. Let $\left(x_{0}, y_{0}\right)$ be an arbitrary initial point in the interior of the first quadrant. Then $\left(0, y_{0}\right) \preceq_{\text {se }}\left(x_{0}, y_{0}\right) \preceq_{\text {se }}\left(x_{0}, 0\right)$ and $T\left(0, y_{0}\right) \preceq_{\mathrm{se}} T\left(x_{0}, y_{0}\right) \preceq_{\mathrm{se}} T\left(x_{0}, 0\right)=E_{\bar{x}}$ and so $T^{n}(0$, $\left.y_{0}\right) \preceq_{\mathrm{se}} T^{n}\left(x_{0}, y_{0}\right) \preceq_{\mathrm{se}} T^{n}\left(x_{0}, 0\right)=E_{\bar{x}}$. In view of Theorems 12 and $14 T^{n}\left(x_{0}, y_{0}\right) \rightarrow E_{\bar{x}}$ or $T^{n}\left(x_{0}, y_{0}\right) \rightarrow E_{0}$ as $n \rightarrow$ $\infty$.

Theorem 16. Assume that $1=4 A_{2} C_{2}$. Then system (8) has two equilibrium points, $E_{\bar{x}}$ which is locally asymptotically stable and $E_{\bar{y}}$ which is nonhyperbolic of the stable type. The singular point $E_{0}$ is global attractor of all points on the $y$-axis, which start below $E_{\bar{y}}$. Furthermore, every point in the interior of the first quadrant below $\mathscr{W}^{s}\left(E_{\bar{y}}\right)$ is attracted to $E_{0}(0,0)$ or $E_{\bar{x}}$ 

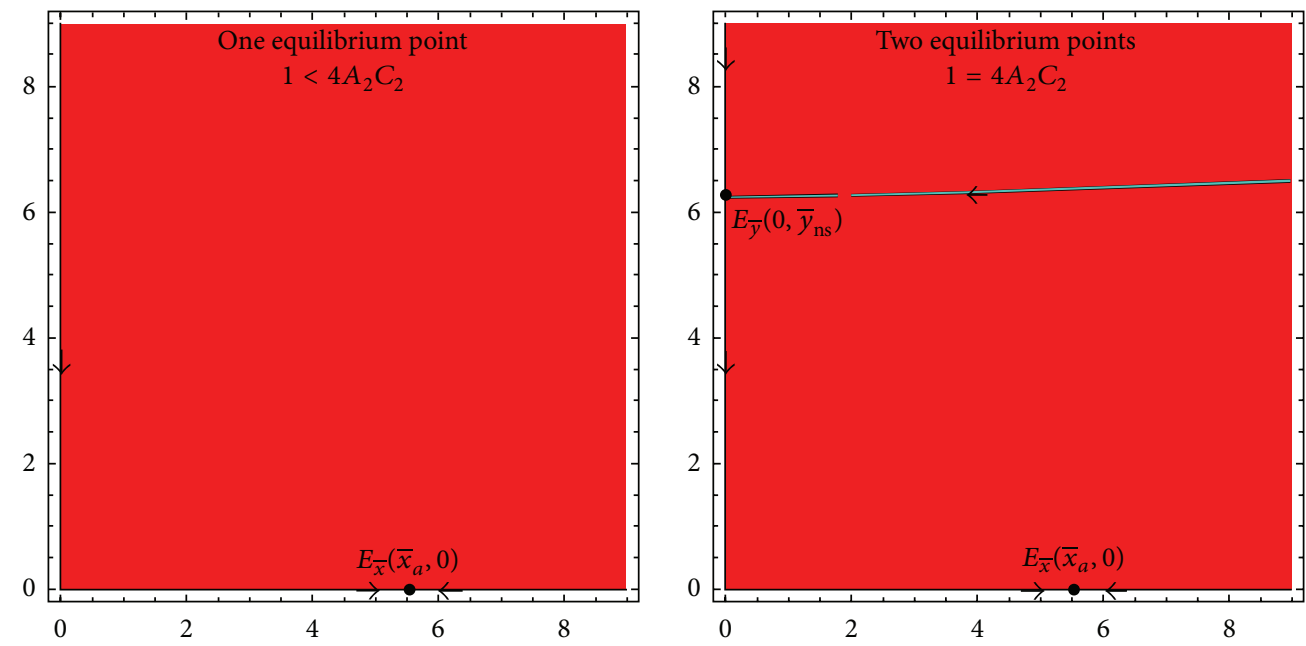

$B_{1}=0.18, C_{1}=0.062, A_{2}=3.25, B_{2}=0.002, C_{2}=0.08$

$B_{1}=0.18, C_{1}=0.062, A_{2}=3.125, B_{2}=0.002, C_{2}=0.08$
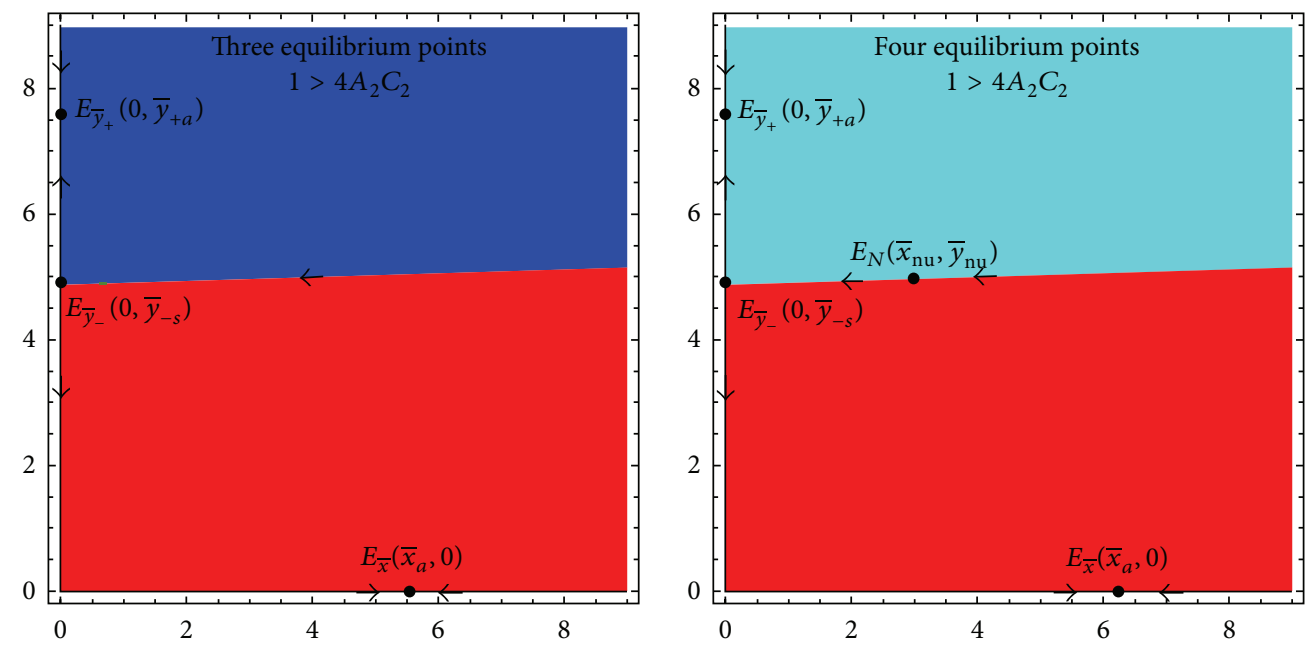

$B_{1}=0.18, C_{1}=0.062, A_{2}=2.98, B_{2}=0.002, C_{2}=0.08$

$B_{1}=116 / 723, C_{1}=15 / 241, A_{2}=1491 / 500, B_{2}=2 / 1000$, $C_{2}=8 / 100$
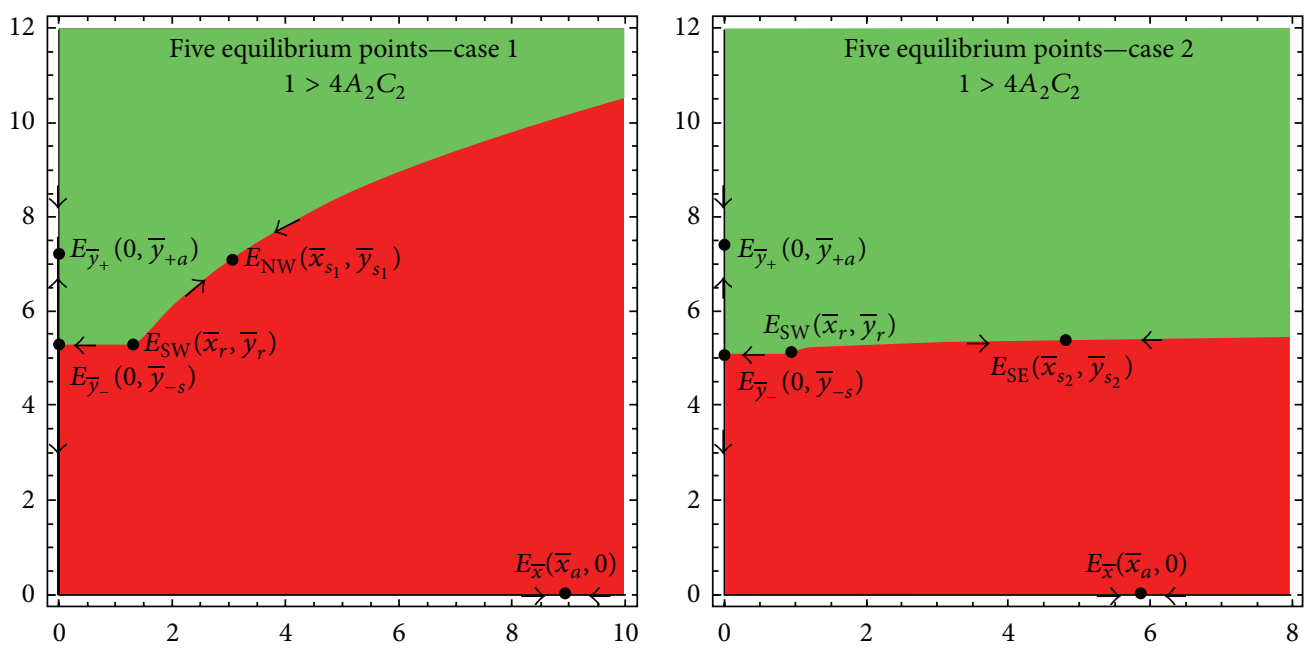

$B_{1}=0.112, C_{1}=0.04, A_{2}=3.05, B_{2}=0.002, C_{2}=0.08$

$B_{1}=0.17, C_{1}=0.03, A_{2}=3.02, B_{2}=0.002, C_{2}=0.08$

FIgURe 3: Global stability. 


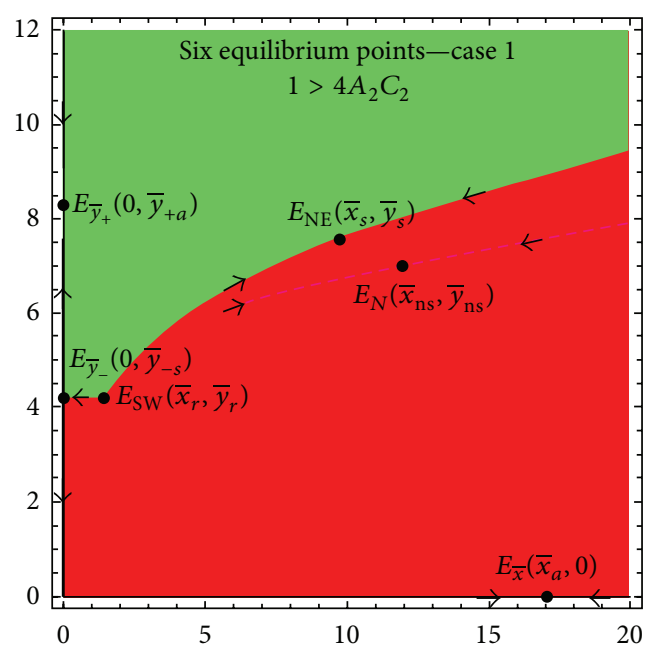

$B_{1}=83 / 1416, C_{1}=30 / 413, A_{2}=349 / 125, B_{2}=2 / 1000$, $C_{2}=8 / 100$

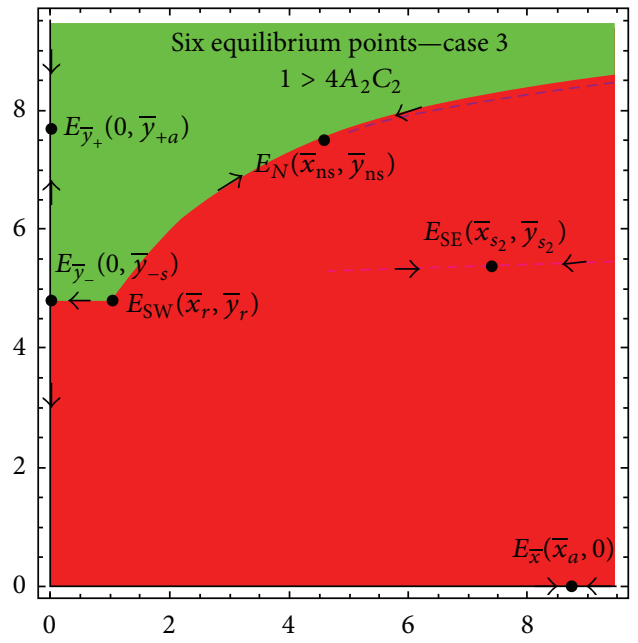

$B_{1}=52165 / 455584, C_{1}=575 / 14856, A_{2}=36971 / 12500$, $B_{2}=2 / 1000, C_{2}=8 / 100$

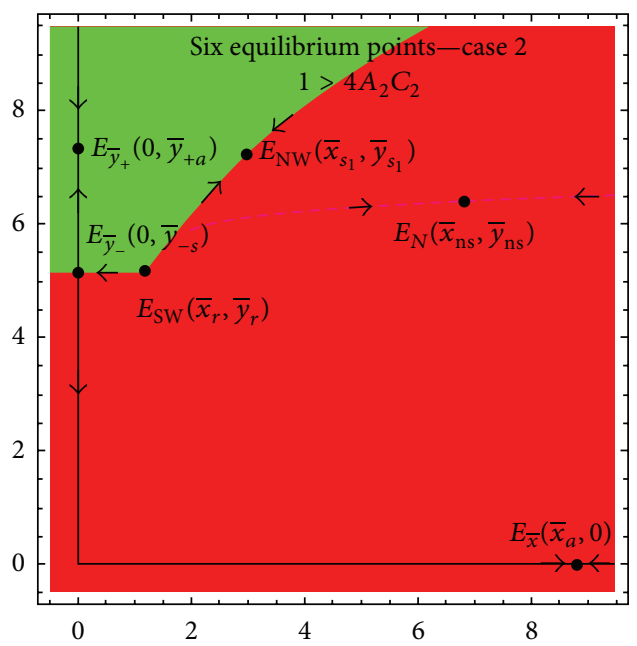

$B_{1}=2045 / 17986, C_{1}=1275 / 33856, A_{2}=9471 / 3125$,

$B_{2}=2 / 1000, C_{2}=8 / 100$

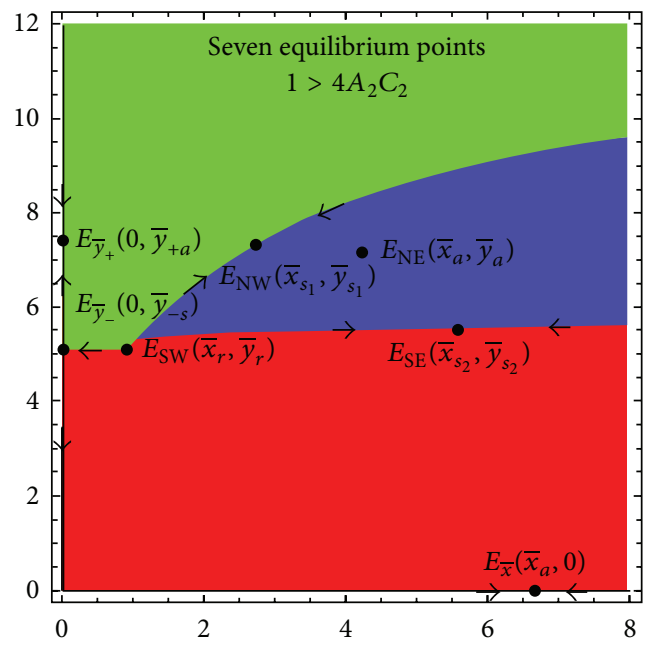

$B_{1}=0.15, C_{1}=0.03, A_{2}=3.02, B_{2}=0.002$,

$C_{2}=0.08$

FIGURE 4: Global stability.

and every point in the first quadrant which starts above $\mathscr{W}^{s}\left(E_{\bar{y}}\right)$ is attracted to $E_{\bar{y}}$.

Proof. Local stability of all equilibrium points follows from Theorem 11. In view of Theorem 12, every solution that starts on the $y$-axis below $E_{\bar{y}}$ converges to 0 in a decreasing manner and every solution that starts on the $x$-axis is equal to $E_{\bar{x}}$ in a single step. In addition, every solution that starts on the $y$-axis above $E_{\bar{y}}$ converges to $E_{\bar{y}}$ in a decreasing way. Let $\left(x_{0}, y_{0}\right)$ be an arbitrary initial point in the interior of the first quadrant below $\mathscr{W}^{s}\left(E_{\bar{y}}\right)$. Then $\left(0, y_{0}\right) \varliminf_{\text {se }}\left(x_{0}, y_{0}\right) \unlhd_{\text {se }}\left(x_{0}, 0\right)$ which implies $T\left(0, y_{0}\right) \unlhd_{\mathrm{se}} T\left(x_{0}, y_{0}\right) \unlhd_{\mathrm{se}} T\left(x_{0}, 0\right)=E_{\bar{x}}$ and so $T^{n}\left(0, y_{0}\right) \unlhd_{\mathrm{se}} T^{n}\left(x_{0}, y_{0}\right) \unlhd_{\mathrm{se}} T^{n}\left(x_{0}, 0\right)=E_{\bar{x}}$. If $y_{0}>\bar{y}$ then $T^{n}\left(x_{0}, y_{0}\right)$ will eventually enter the ordered interval
$I\left(E_{\bar{y}}, E_{\bar{x}}\right)=\{(x, y): 0<x \leq \bar{x}, 0<y \leq \bar{y}\}$. In view of Theorems 12 and $14, T^{n}\left(x_{0}, y_{0}\right) \rightarrow E_{\bar{x}}$ or $T^{n}\left(x_{0}, y_{0}\right) \rightarrow E_{0}$ as $n \rightarrow \infty$.

Now, let $\left(x_{0}, y_{0}\right)$ be an arbitrary initial point in the interior of the first quadrant above $\mathscr{W}^{s}\left(E_{\bar{y}}\right)$. Then $\left(0, y_{0}\right) \preceq_{\text {se }}$ $\left(x_{0}, y_{0}\right) \unlhd_{\text {se }}\left(x_{0}, y_{W}\right)$, where $\left(x_{0}, y_{W}\right) \in \mathscr{W}^{s}\left(E_{\bar{y}}\right)$. This implies $T\left(0, y_{0}\right) \unlhd_{\mathrm{se}} T\left(x_{0}, y_{0}\right) \unlhd_{\mathrm{se}} T\left(x_{0}, y_{W}\right)$ and $\operatorname{so} T^{n}\left(0, y_{0}\right) \unlhd_{\mathrm{se}} T^{n}\left(x_{0}\right.$, $\left.y_{0}\right) \preceq_{\mathrm{se}} T^{n}\left(x_{0}, y_{W}\right)$. Since $T^{n}\left(0, y_{0}\right) \rightarrow E_{\bar{y}}, T\left(x_{0}, y_{W}\right) \rightarrow E_{\bar{y}}$ as $n \rightarrow \infty$, we conclude that $T^{n}\left(x_{0}, y_{0}\right) \rightarrow E_{\bar{y}}$ as $n \rightarrow$ $\infty$.

Theorem 17. Assume that $1>4 A_{2} C_{2}$ and system (8) has three equilibrium points, $E_{\bar{x}}$ and $E_{\bar{y}_{+}}$which are locally asymptotically 
stable and $E_{\bar{y}_{-}}$which is a saddle point. The singular point $E_{0}(0,0)$ is global attractor of all points on $y$-axis, which start below $E_{\bar{y}_{-}}$. The basins of attraction of two equilibrium points are given as

$$
\begin{gathered}
B\left(E_{\bar{y}_{+}}\right)=\left\{\left(x_{0,} y_{0}\right) \text { : points above } \mathscr{W}^{\mathcal{s}}\left(E_{\bar{y}_{-}}\right)\right\}, \\
B\left(E_{\bar{y}_{-}}\right)=\mathscr{W}^{s}\left(E_{\bar{y}_{-}}\right),
\end{gathered}
$$

where $\mathscr{W}^{s}\left(E_{\bar{y}_{-}}\right)$denotes the global stable manifold guaranteed by Theorem 4. Furthermore, every initial point below $\mathscr{W}^{s}\left(E_{\bar{y}_{-}}\right)$ is attracted to $E_{0}(0,0)$ or $E_{\bar{x}}$.

Proof. Local stability of all equilibrium points follows from Theorem 11. The existence of the global stable manifold is guaranteed by Theorem 4 in view of Theorem 13 .

By Theorem 12, every solution that starts on the $y$-axis below $E_{\bar{y}_{-}}$converges to $E_{0}$ in a decreasing manner and every solution that starts on the $x$-axis is equal to $E_{\bar{x}}$ in a single step. In addition, every solution that starts on the $y$-axis above $E_{\bar{y}_{-}}$ converges to $E_{\bar{y}_{+}}$in a monotonic way.

Let $\left(x_{0}, y_{0}\right)^{+}$be an arbitrary initial point in the interior of the first quadrant below $\mathscr{W}^{s}\left(E_{\bar{y}_{-}}\right)$. Then $\left(x_{0}\right.$, $\left.y_{W}\right) \preceq_{\text {se }}\left(x_{0}, y_{0}\right) \preceq_{\text {se }}\left(x_{0}, 0\right)$ which implies $T\left(x_{0}, y_{W}\right) \preceq_{\text {se }} T\left(x_{0}\right.$, $\left.y_{0}\right) \preceq_{\mathrm{se}} T\left(x_{0}, 0\right)=E_{\bar{x}}$ and so $T^{n}\left(x_{0}, y_{W}\right) \preceq_{\mathrm{se}} T^{n}\left(x_{0}, y_{0}\right) \preceq_{\mathrm{se}}$ $T^{n}\left(x_{0}, 0\right)=E_{\bar{x}}$. Since $T^{n}\left(x_{0}, y_{W}\right) \rightarrow E_{\bar{y}_{-}}$as $n \rightarrow \infty$, we conclude that $T^{n}\left(x_{0}, y_{0}\right)$ eventually enters the ordered interval $I\left(E_{\bar{y}_{-}}, E_{\bar{x}}\right)=\left\{(x, y): 0<x \leq \bar{x}, 0<y \leq \bar{y}_{-}\right\}$, in which case it converges to $E_{\bar{x}}$ or $E_{0}(0,0)$.

Finally, let $\left(x_{0}, y_{0}\right)$ be an arbitrary initial point in the interior of the first quadrant above $\mathscr{W}^{s}\left(E_{\bar{y}_{-}}\right)$. Then $\left(0, y_{0}\right) \preceq_{\text {se }}$ $\left(x_{0}, y_{0}\right) \preceq_{\text {se }}\left(x_{0}, y_{W}\right)$, where $\left(x_{0}, y_{W}\right) \in \mathscr{W}^{s}\left(E_{\bar{y}_{-}}\right)$. Thus $T^{n}(0$, $\left.y_{0}\right) \preceq_{\mathrm{se}} T^{n}\left(x_{0}, y_{0}\right) \preceq_{\mathrm{se}} T^{n}\left(x_{0}, y_{W}\right)$, which, by $T^{n}\left(x_{0}, y_{W}\right) \rightarrow$ $E_{\bar{y}_{-}}$as $n \rightarrow \infty$, implies that $T^{n}\left(x_{0}, y_{0}\right)$ eventually lands on the part of $y$-axis above $E_{\bar{y}_{-}}$and so it converges to $E_{\bar{y}_{+}}$.

Theorem 18. Assume that $1>4 A_{2} C_{2}$ and system (8) has four equilibrium points, $E_{\bar{x}}$ and $E_{\bar{y}_{+}}$which are locally asymptotically stable, $E_{\bar{y}_{-}}$which is a saddle point, and $E_{N}$ which is nonhyperbolic of the unstable type. The singular point $E_{0}(0,0)$ is global attractor of all points on the $y$-axis, which start below $E_{\bar{y}_{-}}$. The basins of attraction of three of the equilibrium points are given as

$$
\begin{gathered}
\left\{\left(x_{0}, y_{0}\right): \text { points below } C_{l} \text { such that } x_{0} \geq x_{N}\right\} \subset B\left(E_{\bar{x}}\right), \\
B\left(E_{\bar{y}_{+}}\right)=\left\{\left(x_{0}, y_{0}\right): \text { points above } \mathscr{W}^{s}\left(E_{\bar{y}_{-}}\right) \cup C_{u}\right\}, \\
B\left(E_{N}\right)=\left\{\left(x_{0}, y_{0}\right) \text { : points between } C_{l} \text { and } C_{u}\right\}, \\
B\left(E_{\bar{y}_{-}}\right)=\mathscr{W}^{s}\left(E_{\bar{y}_{-}}\right),
\end{gathered}
$$

where $\mathscr{W}^{s}\left(E_{\bar{y}_{-}}\right)$denotes the global stable manifold guaranteed by Theorem 4 and $C_{l}, C_{u}$ are continuous nondecreasing curves emanating from $E_{N}$, whose existence and properties are guaranteed by Corollary 7. Furthermore, every initial point below $\mathscr{W}^{s}\left(E_{\bar{y}_{-}}\right)$is attracted to $E_{0}(0,0)$ or $E_{\bar{x}}$.
Proof. Local stability of all equilibrium points follows from Theorem 11. The existence of the global stable manifold is guaranteed by Theorems 4 and 13 .

By Theorem 12, every solution that starts on the $y$-axis below $E_{\bar{y}_{-}}$converges to $E_{0}$ in a decreasing manner and every solution that starts on the $x$-axis is equal to $E_{\bar{x}}$ in a single step. In addition, every solution that starts on $y$-axis above $E_{\bar{y}_{-}}$converges to $E_{\bar{y}_{+}}$in a monotonic way.

Let $\left(x_{0}, y_{0}\right)$ be an arbitrary initial point in the interior of the first quadrant below $\mathscr{W}^{s}\left(E_{\bar{y}_{-}}\right) \cup C_{l}$. Assume that $x_{0} \geq \bar{x}_{N}$. Then $\left(x_{0}, y_{W}\right) \preceq_{\text {se }}\left(x_{0}, y_{0}\right) \preceq_{\text {se }}\left(x_{0}, 0\right)$ and so $T\left(x_{0}, y_{W}\right) \preceq_{\mathrm{se}} T\left(x_{0}, y_{0}\right) \preceq_{\mathrm{se}} T\left(x_{0}, 0\right)=E_{\bar{x}}$, where $\left(x_{0}, y_{W}\right) \in$ $C_{l}$ and so $T^{n}\left(x_{0}, y_{W}\right) \preceq_{\mathrm{se}} T^{n}\left(x_{0}, y_{0}\right) \preceq_{\mathrm{se}} T^{n}\left(x_{0}, 0\right)=E_{\bar{x}}$. Since $T^{n}\left(x_{0}, y_{W}\right) \rightarrow E_{N}$ and $T^{n}\left(x_{0}, 0\right) \rightarrow E_{\bar{x}}$ as $n \rightarrow \infty$, we conclude that $T^{n}\left(x_{0}, y_{0}\right)$ eventually enters the ordered interval $I\left(E_{N}, E_{\bar{x}}\right)$, in which case, in view of Corollary 5 , it converges to $E_{\bar{x}}$.

Next, assume that $0<x_{0}<\bar{x}_{N}$. Then $\left(x_{0}, y_{W}\right) \preceq_{\text {se }}\left(x_{0}\right.$, $\left.y_{0}\right) \preceq_{\text {se }}\left(x_{0}, 0\right)$, where $\left(x_{0}, y_{W}\right) \in \mathscr{W}^{s}\left(E_{\bar{y}_{-}}\right)$and so $T\left(x_{0}\right.$, $\left.y_{W}\right) \preceq_{\mathrm{se}} T\left(x_{0}, y_{0}\right) \preceq_{\mathrm{se}} T\left(x_{0}, 0\right)=E_{\bar{x}}$ and so $T^{n}\left(x_{0}, y_{W}\right) \preceq_{\mathrm{se}}$ $T^{n}\left(x_{0}, y_{0}\right) \preceq_{\mathrm{se}} T^{n}\left(x_{0}, 0\right)=E_{\bar{x}}$. Since $T^{n}\left(x_{0}, y_{W}\right) \rightarrow E_{\bar{y}_{-}}$and $T^{n}\left(x_{0}, 0\right) \rightarrow E_{\bar{x}}$ as $n \rightarrow \infty$, we conclude that $T^{n}\left(x_{0}, y_{0}\right)$ eventually enters the ordered interval $I\left(E_{\bar{y}_{-}}, E_{\bar{x}}\right)$, in which case, by Theorems 12 and $14, T^{n}\left(x_{0}, y_{0}\right) \rightarrow E_{\bar{x}}$ or $T^{n}\left(x_{0}\right.$, $\left.y_{0}\right) \rightarrow E_{0}$ as $n \rightarrow \infty$.

Now, let $\left(x_{0}, y_{0}\right)$ be an arbitrary initial point in the interior of the first quadrant above $\mathscr{W}^{s}\left(E_{\bar{y}_{-}}\right) \cup C_{u}$. Assume that $x_{0}>\bar{x}_{N}$. Then $\left(0, y_{0}\right) \preceq_{\text {se }}\left(x_{0}, y_{0}\right) \preceq_{\text {se }}\left(x_{0}, y_{W}\right)$. Assume that $\left(x_{0}, y_{W}\right) \in C_{u}$. Thus $T^{n}\left(0, y_{0}\right) \preceq_{\mathrm{se}} T^{n}\left(x_{0}, y_{0}\right) \preceq_{\mathrm{se}} T^{n}\left(x_{0}, y_{W}\right)$, which by $T^{n}\left(0, y_{0}\right) \rightarrow E_{\bar{y}_{+}}$and $T^{n}\left(x_{0}, y_{W}\right) \rightarrow E_{N}$ as $n \rightarrow \infty$ implies that $T^{n}\left(x_{0}, y_{0}\right)$ eventually enters the ordered interval $I\left(E_{\bar{y}_{+}}, E_{N}\right)$, in which case, in view of Corollary 5 , it converges to $E_{\bar{y}_{+}}$.

Next, assume that $0<x_{0} \leq \bar{x}_{N}$. Then $\left(0, y_{0}\right) \preceq_{\text {se }}\left(x_{0}\right.$, $\left.y_{0}\right) \preceq_{\text {se }}\left(x_{0}, y_{W}\right)$, where $\left(x_{0}, y_{W}\right) \in \mathscr{W}^{s}\left(E_{\bar{y}_{-}}\right)$and so $T^{n}(0$, $\left.y_{0}\right) \preceq_{\mathrm{se}} T^{n}\left(x_{0}, y_{0}\right) \preceq_{\mathrm{se}} T^{n}\left(x_{0}, y_{W}\right)$. Since $T^{n}\left(x_{0}, y_{W}\right) \rightarrow E_{\bar{y}_{-}}$ and $T^{n}\left(0, y_{0}\right) \rightarrow E_{\bar{y}_{+}}$as $n \rightarrow \infty$, we conclude that $T^{n}\left(x_{0}, y_{0}\right)$ converges to $E_{\bar{y}_{+}}$.

Finally, let $\left(x_{0}, y_{0}\right)$ be an arbitrary initial point between $C_{l}$ and $C_{u}$. Then $T^{n}\left(x_{0}, y_{0}\right)$ stays between $C_{l}$ and $C_{u}$ for all $n$ and in view of Corollary 7 it must converge to $E_{N}$.

Conjecture 19. Based on our numerical simulations we believe that $C_{l}=C_{u}$ in Theorem 18 .

Theorem 20. Assume that $1>4 A_{2} C_{2}$ and system (8) has five equilibrium points, $E_{\bar{x}}, E_{\bar{y}_{+}}$which are locally asymptotically stable, $E_{\bar{y}_{-}}$and $E_{N W}$ (resp., $E_{S E}$ ) which are saddle points, and $E_{S W}$ which is a repeller. The singular point $E_{0}(0,0)$ is global attractor of all points on the $y$-axis, which start below $E_{\bar{y}_{-}}$. The basins of attraction of four of the equilibrium points are given as

$$
\begin{aligned}
& \left\{\left(x_{0}, y_{0}\right): \text { points below } \mathscr{W}^{s}\left(E_{N W}\right)\right\} \subset B\left(E_{\bar{x}}\right), \\
& B\left(E_{\bar{y}_{+}}\right) \\
= & \left\{\left(x_{0}, y_{0}\right) \text { : points above } \mathscr{W}^{s}\left(E_{\bar{y}_{-}}\right) \cup \mathscr{W}^{s}\left(E_{N W}\right)\right\},
\end{aligned}
$$




$$
\begin{gathered}
B\left(E_{N W}\right)=\mathscr{W}^{s}\left(E_{N W}\right), \\
B\left(E_{\bar{y}_{-}}\right)=\mathscr{W}^{s}\left(E_{\bar{y}_{-}}\right),
\end{gathered}
$$

where $\mathscr{W}^{s}\left(E_{\bar{y}_{-}}\right)$and $\mathscr{W}^{s}\left(E_{N W}\right)$ denote the global stable manifolds whose existence is guaranteed by Theorem 4. Furthermore, every initial point below $\mathscr{W}^{s}\left(E_{\bar{y}_{-}}\right)$is attracted to $E_{0}$ or $E_{\bar{y}}$.

Proof. Local stability of all equilibrium points follows from Theorem 11. We present the proof in the case of the equilibrium point $E_{\mathrm{NW}}$. The proof in the case of the equilibrium point $E_{\mathrm{SE}}$ is similar.

The existence of the global stable manifold is guaranteed by Theorems 4 and 13 .

By Theorem 12 , every solution that starts on the $y$-axis below $E_{\bar{y}_{-}}$converges to $E_{0}$ in a decreasing manner and every solution that starts on the $x$-axis is equal to $E_{\bar{x}}$ in a single step. In addition, every solution that starts on the $y$-axis above $E_{\bar{y}_{-}}$ converges to $E_{\bar{y}_{+}}$in a monotonic way.

Let $\left(x_{0}, y_{0}\right)$ be an arbitrary initial point in the interior of the first quadrant below $\mathscr{W}^{s}\left(E_{\bar{y}_{-}}\right) \cup \mathscr{W}^{s}\left(E_{\mathrm{NW}}\right)$. Assume that $x_{0}>\bar{x}_{\mathrm{SW}}$. Then $\left(x_{0}, y_{W}\right) \preceq_{\mathrm{se}}\left(x_{0}, y_{0}\right) \preceq_{\mathrm{se}}\left(x_{0}, 0\right)$ which implies $T\left(x_{0}, y_{W}\right) \preceq_{\mathrm{se}} T\left(x_{0}, y_{0}\right) \preceq_{\mathrm{se}} T\left(x_{0}, 0\right)=E_{\bar{x}}$, where $\left(x_{0}, y_{W}\right) \in$ $\mathscr{W}^{s}\left(E_{\mathrm{NW}}\right)$ and so $T^{n}\left(x_{0}, y_{W}\right) \preceq_{\mathrm{se}} T^{n}\left(x_{0}, y_{0}\right) \preceq_{\mathrm{se}} T^{n}\left(x_{0}, 0\right)=$ $E_{\bar{x}}$. Since $T^{n}\left(x_{0}, y_{W}\right) \rightarrow E_{\mathrm{NW}}$ and $T^{n}\left(x_{0}, 0\right) \rightarrow E_{\bar{x}}$ as $n \rightarrow$ $\infty$, we conclude that $T^{n}\left(x_{0}, y_{0}\right)$ eventually enters the ordered interval $I\left(E_{\mathrm{NW}}, E_{\bar{x}}\right)$, in which case, in view of Corollary 5 , it converges to $E_{\bar{x}}$.

Next, assume that $0<x_{0} \leq \bar{x}_{\mathrm{SW}}$. Then $\left(x_{0}, y_{W}\right) \preceq_{\mathrm{se}}\left(x_{0}\right.$, $\left.y_{0}\right) \preceq_{\text {se }}\left(x_{0}, 0\right)$, where $\left(x_{0}, y_{W}\right) \in \mathscr{W}^{s}\left(E_{\bar{y}_{-}}\right)$. Thus $T\left(x_{0}, y_{W}\right) \preceq_{\text {se }}$ $T\left(x_{0}, y_{0}\right) \preceq_{\mathrm{se}} T\left(x_{0}, 0\right)=E_{\bar{x}}$ and so $T^{n}\left(x_{0}, y_{W}\right) \preceq_{\mathrm{se}} T^{n}\left(x_{0}\right.$, $\left.y_{0}\right) \preceq_{\mathrm{se}} T^{n}\left(x_{0}, 0\right)=E_{\bar{x}}$. Since $T^{n}\left(x_{0}, y_{W}\right) \rightarrow E_{\bar{y}_{-}}$and $T^{n}\left(x_{0}\right.$, $0) \rightarrow E_{\bar{x}}$ as $n \rightarrow \infty$, we conclude that $T^{n}\left(x_{0}, y_{0}\right)$ eventually enters the interior of the ordered interval $I\left(E_{\bar{y}_{-}}, E_{\bar{x}}\right)$, in which case, it converges to $E_{0}$ or $E_{\bar{x}}$.

Now, let $\left(x_{0}, y_{0}\right)$ be an arbitrary initial point in the interior of the first quadrant above $\mathscr{W}^{s}\left(E_{\bar{y}_{-}}\right) \cup \mathscr{W}^{s}\left(E_{\mathrm{NW}}\right)$. Assume $x_{0}>$ $\bar{x}_{\mathrm{SW}}$. Then $\left(0, y_{0}\right) \preceq_{\mathrm{se}}\left(x_{0}, y_{0}\right) \preceq_{\mathrm{se}}\left(x_{0}, y_{W}\right)$, where $\left(x_{0}, y_{W}\right) \in$ $\mathscr{W}^{s}\left(E_{\mathrm{NW}}\right)$ and so $T^{n}\left(0, y_{0}\right) \preceq_{\mathrm{se}} T^{n}\left(x_{0}, y_{0}\right) \preceq_{\mathrm{se}} T^{n}\left(x_{0}, y_{W}\right)$. Since $T^{n}\left(0, y_{0}\right) \rightarrow E_{\bar{y}_{+}}$and $T^{n}\left(x_{0}, y_{W}\right) \rightarrow E_{\mathrm{NW}}$ as $n \rightarrow \infty$, then $T^{n}\left(x_{0}, y_{0}\right)$ eventually enters the ordered interval $I\left(E_{\bar{y}_{+}}\right.$, $\left.E_{\mathrm{NW}}\right)$, in which case, in view of Corollary 5 , it converges to $E_{\bar{y}_{+}}$.

Next, assume that $0<x_{0} \leq \bar{x}_{\mathrm{SW}}$. Then $\left(0, y_{0}\right) \preceq_{\mathrm{se}}\left(x_{0}\right.$, $\left.y_{0}\right) \preceq_{\text {se }}\left(x_{0}, y_{W}\right)$, where $\left(x_{0}, y_{W}\right) \in \mathscr{W}^{s}\left(E_{\bar{y}_{-}}\right)$and so $T^{n}(0$, $\left.y_{0}\right) \preceq_{\mathrm{se}} T^{n}\left(x_{0}, y_{0}\right) \preceq_{\mathrm{se}} T^{n}\left(x_{0}, y_{W}\right)$. Since $T^{n}\left(x_{0}, y_{W}\right) \rightarrow E_{\bar{y}_{-}}$ and $T^{n}\left(0, y_{0}\right) \rightarrow E_{\bar{y}_{+}}$as $n \rightarrow \infty$, we conclude that $T^{n}\left(x_{0}, y_{0}\right)$ converges to $E_{\bar{y}_{+}}$.

Theorem 21. Assume that $1>4 A_{2} C_{2}$ and system (8) has six equilibrium points, $E_{\bar{x}}, E_{\bar{y}_{+}}$which are locally asymptotically stable, $E_{\bar{y}}$ and $E_{N E}$ (resp., $E_{S E}$ or $E_{N W}$ ) which are saddle points, $E_{S W}$ which is a repeller, and $E_{N}$ which is nonhyperbolic of the stable type. The singular point $E_{0}(0,0)$ is global attractor of all points on the $y$-axis, which start below $E_{\bar{y}_{-}}$. The basins of attraction of five of the equilibrium points are given as

$$
\begin{gathered}
\left\{\left(x_{0}, y_{0}\right) \text { : points below } \mathscr{W}^{s}\left(E_{N}\right)\right\} \subset B\left(E_{\bar{x}}\right), \\
B\left(E_{\bar{y}_{+}}\right) \\
=\left\{\left(x_{0}, y_{0}\right) \text { : points above } \mathscr{W}^{s}\left(E_{\bar{y}_{-}}\right) \cup \mathscr{W}^{s}\left(E_{N E}\right)\right\}, \\
B\left(E_{N}\right) \\
=\left\{\left(x_{0}, y_{0}\right) \text { : region bounded by } \mathscr{W}^{s}\left(E_{N}\right) \text { and } \mathscr{W}^{s}\left(E_{N E}\right)\right\}, \\
B\left(E_{\bar{y}_{-}}\right)=\mathscr{W}^{s}\left(E_{\bar{y}_{-}}\right), \\
B\left(E_{N E}\right)=\mathscr{W}^{s}\left(E_{N E}\right),
\end{gathered}
$$

where $\mathscr{W}^{s}\left(E_{\bar{y}_{-}}\right), \mathscr{W}^{s}\left(E_{N}\right)$, and $\mathscr{W}^{s}\left(E_{N E}\right)$ denote the global stable manifolds whose existence is guaranteed by Theorem 4. Furthermore, every initial point below $\mathscr{W}^{s}\left(E_{\bar{y}_{-}}\right)$is attracted to $E_{0}$ or $E_{\bar{x}}$.

Proof. Local stability of all equilibrium points follows from Theorem 11. We present the proof in the case of the equilibrium point $E_{\mathrm{NE}}$. The proof in the case of the equilibrium points $E_{\mathrm{SE}}$ and $E_{\mathrm{NW}}$ is similar.

The existence of the global stable manifolds are guaranteed by Theorems 4 and 13 .

The proofs of the basins of attractions $B\left(E_{\bar{x}}\right), B\left(E_{\bar{y}_{+}}\right)$ are the same as the proofs for the corresponding basins of attraction in Theorem 20, so we will only give the proof for $B\left(E_{N}\right)$. Indeed, $B\left(E_{N}\right)$ is an invariant set and $T^{n}\left(B\left(E_{N}\right)\right)$ is a subset of the interior of the ordered interval $I\left(E_{\mathrm{NE}}, E_{N}\right)$ for $n$ large. In view of Corollary 5 the interior of the ordered interval $I\left(E_{\mathrm{NE}}, E_{N}\right)$ is attracted to $E_{N}$.

Theorem 22. Assume that $1>4 A_{2} C_{2}$ and system (8) has seven equilibrium points, $E_{\bar{x}}, E_{\bar{y}_{+}}, E_{N E}$ which are locally asymptotically stable, $E_{\bar{y}_{-}}, E_{S E}, E_{N W}$ which are saddle points, and $E_{S W}$ which is a repeller. The singular point $E_{0}(0,0)$ is global attractor of all points on $y$-axis, which start below $E_{\bar{y}_{-}}$. The basins of attraction of six of the equilibrium points are given as

$$
\begin{gathered}
\left\{\left(x_{0}, y_{0}\right) \text { : points below } \mathscr{W}^{s}\left(E_{S E}\right)\right\} \subset B\left(E_{\bar{x}}\right), \\
B\left(E_{\bar{y}_{+}}\right) \\
=\left\{\left(x_{0}, y_{0}\right) \text { : points above } \mathscr{W}^{s}\left(E_{\bar{y}_{-}}\right) \cup \mathscr{W}^{s}\left(E_{N W}\right)\right\}, \\
B\left(E_{N E}\right) \\
=\left\{\left(x_{0}, y_{0}\right) \text { : region bounded by } \mathscr{W}^{s}\left(E_{S E}\right) \text { and } \mathscr{W}^{s}\left(E_{N W}\right)\right\}, \\
B\left(E_{\bar{y}_{-}}\right)=\mathscr{W}^{s}\left(E_{\bar{y}_{-}}\right), \\
B\left(E_{S E}\right)=\mathscr{W}^{s}\left(E_{S E}\right), \\
B\left(E_{N W}\right)=\mathscr{W}^{s}\left(E_{N W}\right),
\end{gathered}
$$


where $\mathscr{W}^{s}\left(E_{\bar{y}_{-}}\right), \mathscr{W}^{s}\left(E_{N W}\right)$, and $\mathscr{W}^{s}\left(E_{S E}\right)$ denote the global stable manifolds whose existence is guaranteed by Theorem 4. Furthermore, every initial point below $\mathscr{W}^{s}\left(E_{\bar{y}_{-}}\right)$is attracted to $E_{0}$ or $E_{\bar{x}}$.

Proof. Local stability of all equilibrium points follows from Theorem 11. Proofs of the basins of attractions $B\left(E_{\bar{x}}\right), B\left(E_{\bar{y}_{+}}\right)$ are the same as the proofs for corresponding basins of attraction in Theorem 20. So we only give the proof for $B\left(E_{\mathrm{NE}}\right)$. Indeed, $B\left(E_{\mathrm{NE}}\right)$ is an invariant set and $T^{n}\left(B\left(E_{\mathrm{NE}}\right)\right)$ is a subset of the interior of the ordered interval $I\left(E_{\mathrm{NW}}, E_{\mathrm{SE}}\right)$ for $n$ large. In view of Corollary 5 the interior of the ordered interval $\left(E_{\mathrm{NW}}, E_{\mathrm{SE}}\right)$ is attracted to $E_{\mathrm{NE}}$.

\section{Conflict of Interests}

The authors declare that there is no conflict of interests regarding the publication of this paper.

\section{References}

[1] M. R. S. Kulenovic and G. Ladas, Dynamics of Second Order Rational Difference Equations, Chapman \& Hall/CRC, Boca Raton, Fla, USA, 2001.

[2] M. R. S. Kulenovic and O. Merino, Disrete Dynamical Systems and Difference Equations with Mathematica, Chapman \& Hall/CRC Press, Boca Raton, Fla, USA, 2002.

[3] H. R. Thieme, Mathematics in Population Biology, Princeton Series in Theoretical and Computational Biology, Princeton University Press, Princeton, NJ, USA, 2003.

[4] G. G. Thomson, "A proposal for a threshold stock size and maximum fishing mortality rate," in Risk Evaluation and Biological Reference Points for Fisheries Management, S. J. Smith, J. J. Hunt, and D. Rivard, Eds., vol. 120, pp. 303-320, Canadian Special Publication of Fisheries and Aquatic Sciences, 1993.

[5] A. J. Harry, C. M. Kent, and V. L. Kocic, "Global behavior of solutions of a periodically forced Sigmoid Beverton-Holt model," Journal of Biological Dynamics, vol. 6, no. 2, pp. 212234, 2012.

[6] J. M. Cushing, S. Levarge, N. Chitnis, and S. M. Henson, "Some discrete competition models and the competitive exclusion principle," Journal of Difference Equations and Applications, vol. 10, no. 13-15, pp. 1139-1151, 2004.

[7] M. R. S. Kulenović and O. Merino, "Global bifurcation for discrete competitive systems in the plane," Discrete and Continuous Dynamical Systems. Series B. A Journal Bridging Mathematics and Sciences, vol. 12, no. 1, pp. 133-149, 2009.

[8] D. Clark and M. R. S. Kulenović, "A coupled system of rational difference equations," Computers \& Mathematics with Applications, vol. 43, no. 6-7, pp. 849-867, 2002.

[9] D. Clark, M. R. Kulenović, and J. F. Selgrade, "Global asymptotic behavior of a two-dimensional difference equation modelling competition," Nonlinear Analysis. Theory, Methods \& Applications, vol. 52, no. 7, pp. 1765-1776, 2003.

[10] S. Kalabušić, M. R. S. Kulenović, and E. Pilav, "Dynamics of a two-dimensional system of rational difference equations of Leslie-Gower type," Advances in Difference Equations, vol. 2011, article 29, 2011.

[11] S. Kalabušić, M. R. Kulenović, and E. Pilav, "Multiple attractors for a competitive system of rational difference equations in the plane," Abstract and Applied Analysis, vol. 2011, Article ID 295308, 35 pages, 2011.

[12] M. R. S. Kulenović, O. Merino, and M. Nurkanović, "Global dynamics of certain competitive system in the plane," Journal of Difference Equations and Applications, vol. 18, no. 12, pp. 19511966, 2012.

[13] M. R. S. Kulenović and M. Nurkanović, "Asymptotic behavior of a system of linear fractional difference equations," Journal of Inequalities and Applications, no. 2, pp. 127-143, 2005.

[14] M. R. S. Kulenović and M. Nurkanović, "Asymptotic behavior of a competitive system of linear fractional difference equations," Advances in Difference Equations, vol. 2006, Article ID 19756, 13 pages, 2006.

[15] S. Basu and O. Merino, "On the global behavior of solutions to a planar system of difference equations," Communications on Applied Nonlinear Analysis, vol. 16, no. 1, pp. 89-101, 2009.

[16] A. Brett and M. R. S. Kulenović, "Basins of attraction of equilibrium points of monotone difference equations," Sarajevo Journal of Mathematics, vol. 5, no. 2, pp. 211-233, 2009.

[17] D. Burgić, S. Kalabuvić, and M. R. S. Kulenović, "Nonhyperbolic dynamics for competitive systems in the plane and global period-doubling bifurcations," Advances in Dynamical Systems and Applications, vol. 3, no. 2, pp. 229-249, 2008.

[18] M. R. S. Kulenović and O. Merino, "Invariant manifolds for competitive discrete systems in the plane," International Journal of Bifurcation and Chaos, vol. 20, no. 8, pp. 2471-2486, 2010.

[19] Y. Kang and H. L. Smith, "Global dynamics of a discrete twospecies lottery-Ricker competition model," Journal of Biological Dynamics, vol. 6, no. 2, pp. 358-376, 2012.

[20] G. Livadiotis and S. Elaydi, "General Allee effect in two-species population biology," Journal of Biological Dynamics, vol. 6, no. 2, pp. 959-973, 2012.

[21] G. Livadiotis, L. Assas, S. Elaydi, E. Kwessi, and D. Ribble, "Competition models with Allee effects," Journal of Difference Equations and Applications, vol. 20, no. 8, pp. 1127-1151, 2014.

[22] Y. Chow and S. R. Jang, "Multiple attractors in a Leslie-Gower competition system with Allee effects," Journal of Difference Equations and Applications, vol. 20, no. 2, pp. 169-187, 2014.

[23] J. E. Franke and A.-A. Yakubu, "Mutual exclusion versus coexistence for discrete competitive systems," Journal of Mathematical Biology, vol. 30, no. 2, pp. 161-168, 1991.

[24] J. E. Franke and A.-A. Yakubu, "Global attractors in competitive systems," Nonlinear Analysis: Theory, Methods \& Applications, vol. 16, no. 2, pp. 111-129, 1991.

[25] J. E. Franke and A.-A. Yakubu, "Geometry of exclusion principles in discrete systems," Journal of Mathematical Analysis and Applications, vol. 168, no. 2, pp. 385-400, 1992.

[26] M. P. Hassell and H. N. Comins, "Discrete time models for twospecies competition," Theoretical Population Biology, vol. 9, no. 2, pp. 202-221, 1976.

[27] M. Hirsch and H. Smith, "Monotone dynamical systems," in Handbook of Differential Equations: Ordinary Differential Equations, vol. 2, pp. 239-357, Elsevier B. V., Amsterdam, The Netherlands, 2005.

[28] M. W. Hirsch and H. Smith, "Monotone maps: a review," Journal of Difference Equations and Applications, vol. 11, no. 4-5, pp. 379398, 2005.

[29] H. Jiang and T. D. Rogers, “The discrete dynamics of symmetric competition in the plane," Journal of Mathematical Biology, vol. 25, no. 6, pp. 573-596, 1987. 
[30] W. Krawcewicz and T. D. Rogers, "Perfect harmony: the discrete dynamics of cooperation," Journal of Mathematical Biology, vol. 28, no. 4, pp. 383-410, 1990.

[31] M. R. S. Kulenović and O. Merino, "Competitive-exclusion versus competitive-coexistence for systems in the plane," Discrete and Continuous Dynamical Systems, Series B, vol. 6, no. 5, pp. 1141-1156, 2006.

[32] M. R. S. Kulenović and M. Nurkanović, "Global asymptotic behavior of a two-dimensional system of difference equations modeling cooperation," Journal of Difference Equations and Applications, vol. 9, no. 1, pp. 149-159, 2003.

[33] R. M. May, Stability and Complexity in Model Ecosystems, Princeton University Press, Princeton, NJ, USA, 2001.

[34] H. L. Smith, "Periodic competitive differential equations and the discrete dynamics of competitive maps," Journal of Differential Equations, vol. 64, no. 2, pp. 165-194, 1986.

[35] H. L. Smith, "Planar competitive and cooperative difference equations," Journal of Difference Equations and Applications, vol. 3, no. 5-6, pp. 335-357, 1998.

[36] A.-A. Yakubu, "The effects of planting and harvesting on endangered species in discrete competitive systems," Mathematical Biosciences, vol. 126, no. 1, pp. 1-20, 1995.

[37] A.-A. Yakubu, "A discrete competitive system with planting," Journal of Difference Equations and Applications, vol. 4, pp. 213214, 1998.

[38] P. de Mottoni and A. Schiaffino, "Competition systems with periodic coefficients: a geometric approach," Journal of Mathematical Biology, vol. 11, no. 3, pp. 319-335, 1981.

[39] P. Hess, Periodic-Parabolic Boundary Value Problems and Positivity, vol. 247 of Pitman Research Notes in Mathematics Series, Longman Scientific \& Technical, Harlow, UK, 1991.

[40] E. Camouzis, M. R. S. Kulenović, G. Ladas, and O. Merino, "Rational systems in the plane," Journal of Difference Equations and Applications, vol. 15, no. 3, pp. 303-323, 2009.

[41] L. Yang, X. Hou, and Z. Zeng, "A complete discrimination system for polynomials," Science in China. Series E. Technological Sciences, vol. 39, no. 6, pp. 628-646, 1996. 


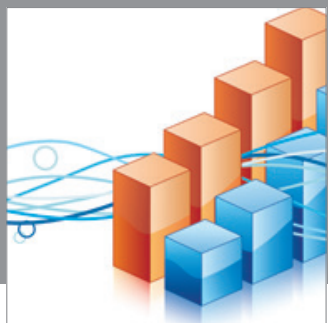

Advances in

Operations Research

mansans

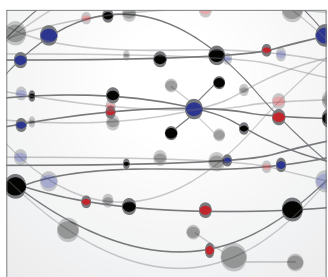

The Scientific World Journal
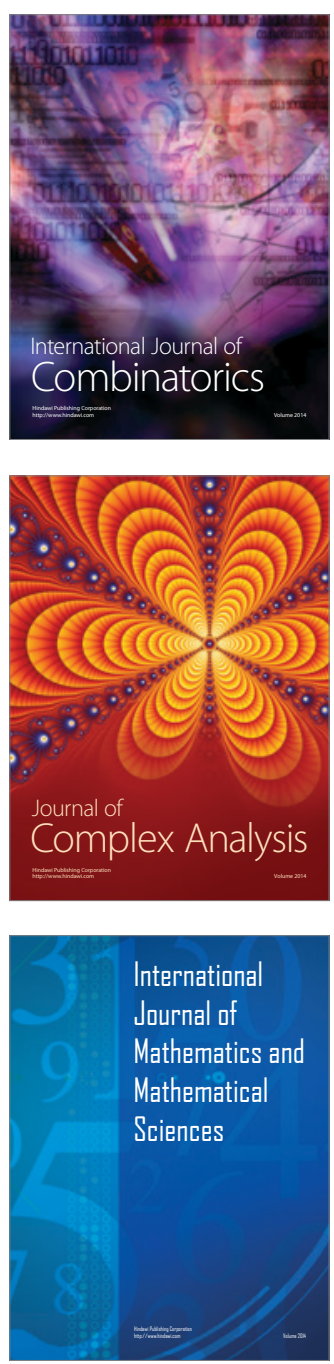
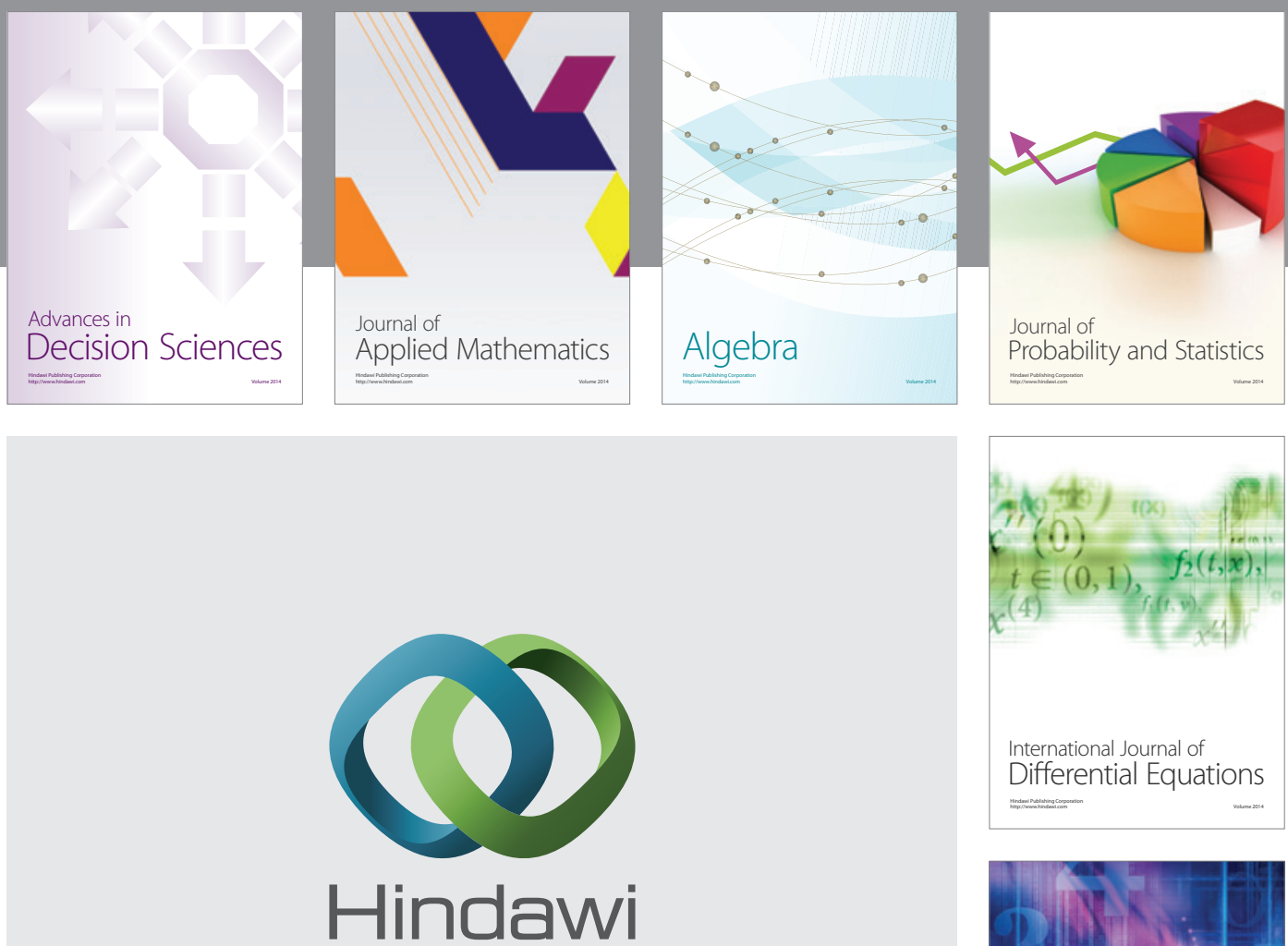

Submit your manuscripts at http://www.hindawi.com
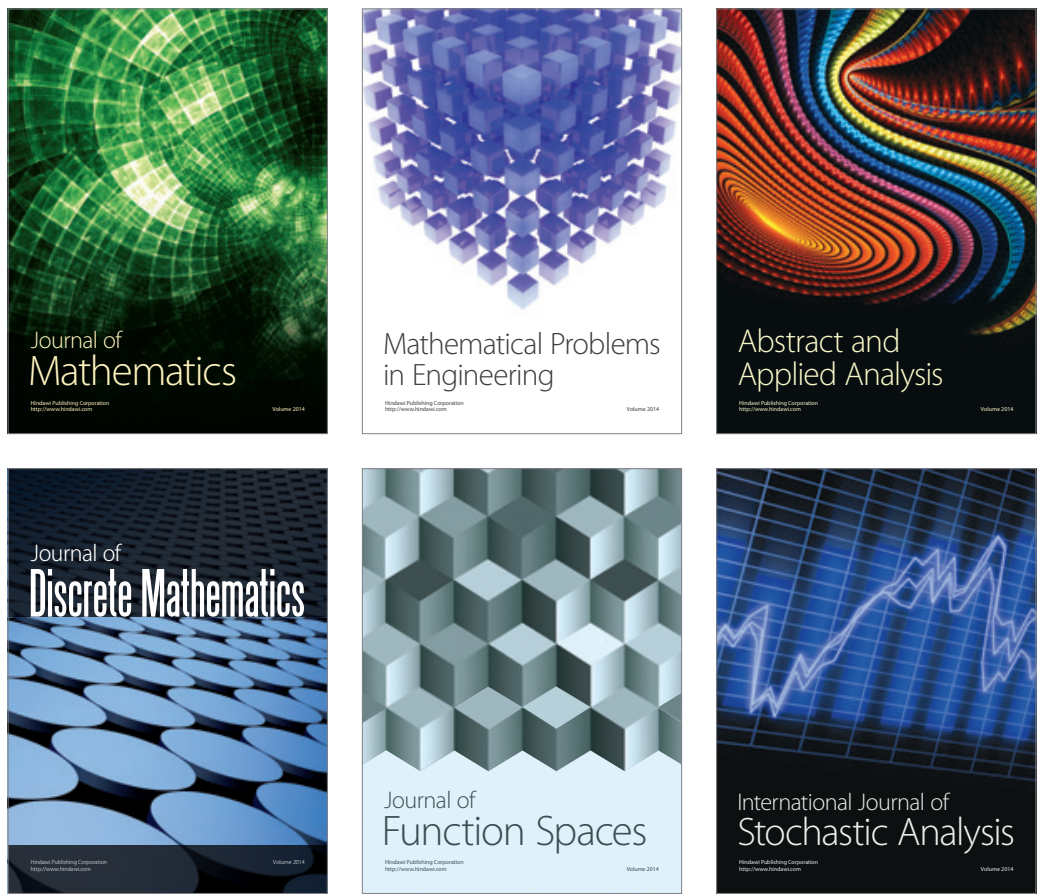

Journal of

Function Spaces

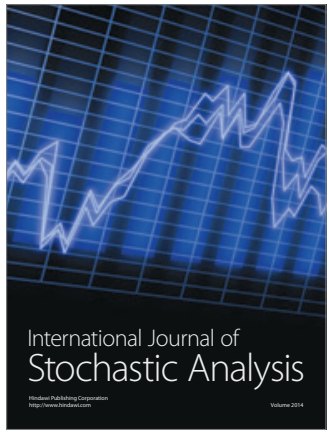

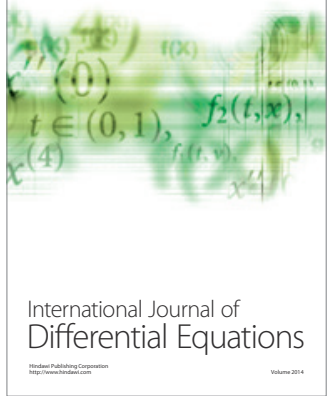
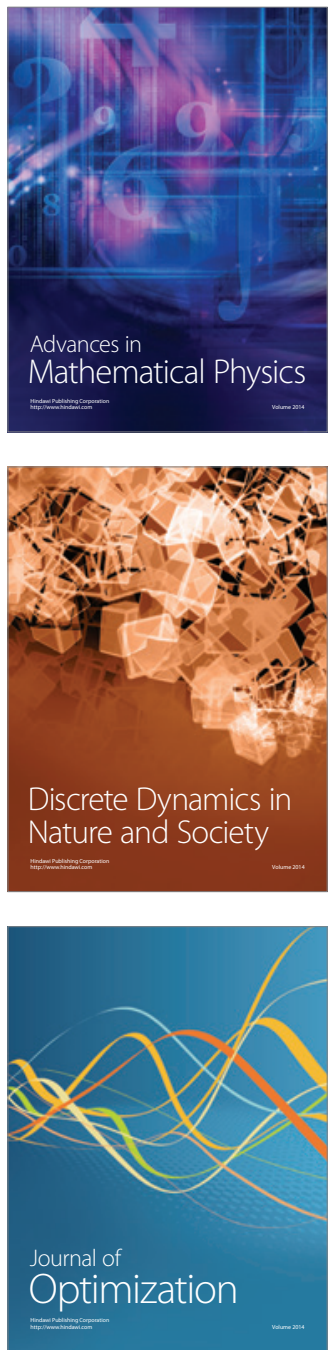